\title{
Expert's Choice: 2018's Most Exciting Research in the Field of Pediatric Exercise Science
}

\author{
Alon Eliakim, ${ }^{1}$ Bareket Falk, ${ }^{2}$ Neil Armstrong, ${ }^{3}$ Fátima Baptista, ${ }^{4}$ David G. Behm, ${ }^{5}$ Nitzan Dror, ${ }^{6}$ \\ Avery D. Faigenbaum, ${ }^{7}$ Kathleen F. Janz, ${ }^{8}$ Jaak Jürimäe, ${ }^{9}$ Amanda L. McGowan, ${ }^{10}$ Dan Nemet, ${ }^{1}$ \\ Paolo T. Pianosi, ${ }^{11}$ Matthew B. Pontifex, ${ }^{10}$ Shlomit Radom-Aizik, ${ }^{6}$ Thomas Rowland, ${ }^{12}$ \\ and Alex V. Rowlands ${ }^{13}$ \\ ${ }^{1}$ Meir Medical Center, Tel-Aviv University; ${ }^{2}$ Brock University; ${ }^{3}$ University of Exeter; ${ }^{4}$ Universidade de Lisboa; \\ ${ }^{5}$ Memorial University of Newfoundland; ${ }^{6}$ University of California Irvine; ${ }^{7}$ The College of New Jersey; \\ ${ }^{8}$ University of lowa; ${ }^{9}$ University of Tartu; ${ }^{10}$ Michigan State University; ${ }^{11}$ University of Minnesota; \\ ${ }^{12}$ Skidmore College; ${ }^{13}$ University of Leicester
}

\begin{abstract}
This commentary highlights 23 noteworthy publications from 2018, selected by leading scientists in pediatric exercise science. These publications have been deemed as significant or exciting in the field as they (a) reveal a new mechanism, (b) highlight a new measurement tool, (c) discuss a new concept or interpretation/application of an existing concept, or (d) describe a new therapeutic approach or clinical tool in youth. In some cases, findings in adults are highlighted, as they may have important implications in youth. The selected publications span the field of pediatric exercise science, specifically focusing on: aerobic exercise and training; neuromuscular physiology, exercise, and training; endocrinology and exercise; resistance training; physical activity and bone strength; growth, maturation, and exercise; physical activity and cognition; childhood obesity, physical activity, and exercise; pulmonary physiology or diseases, exercise, and training; immunology and exercise; cardiovascular physiology and disease; and physical activity, inactivity, and health.
\end{abstract}

Keywords: youth exercise, pediatric health, youth fitness

\section{Introduction}

The Expert's Choice section aims to highlight the most significant or exciting papers in specific areas of pediatric exercise science, published in the preceding year (2018). A "significant" or "exciting" publication is one that either: (a) reveals a new mechanism, (b) highlights a new measurement tool, (c) discusses a new concept or provides a new interpretation or application of an existing concept, or (d) describes a new therapeutic approach or clinical tool.

For each specific area, a leading scientist was asked to select 1 to 2 noteworthy publications. The selected publications' abstracts (where available) are included for reference, followed by the

Eliakim and Nemet are with Meir Medical Center, Tel-Aviv University, Israel. Falk is the Editor-in-Chief of Pediatric Exercise Science and is with Brock University, St. Catharines, Ontario, Canada. Armstrong is with University of Exeter, Exeter, UK. Baptista is with Universidade de Lisboa, Lisbon, Portugal. Behm is with Memorial University of Newfoundland, St. John's, Newfoundland, Canada. Dror and Radom-Aizik are with University of California Irvine, Irvine, CA. Faigenbaum is with The College of New Jersey, Ewing, NJ, USA. Janz is with University of Iowa, Iowa City, IA, USA. Jürimäe is with University of Tartu, Tartu, Estonia. McGowan and Pontifex are with Michigan State University, East Lansing, MI, USA. Pianosi is with University of Minnesota, Minneapolis, MN, USA. Rowland is with Skidmore College, Saratoga Springs, NY, USA. Rowlands is with University of Leicester, Leicester, UK. Falk (bfalk@brocku.ca) is corresponding author. expert's summary of the publication and commentary on the reasons for the selection. These scientists were also asked to provide their insights of the knowledge gaps in the respective area and to suggest future research directions. The result is a set of relatively short, informative, insightful, and sometimes provocative commentaries.

As expected, most of the highlighted publications focus on the pediatric population. However, interestingly, some commentaries highlight studies of adults, the findings of which can provide much-needed insight for the role of exercise during youth. An overall theme in many of the commentaries is the revelation of the scant information available in specific areas in youth. Several studies were highlighted because of their methodological approach, emphasizing the methodological challenges presented in pediatric research.

This year, all commentaries were combined in a single paper, with the subject areas (and associated choices) presented in alphabetical order by the subject area expert's surname. The commentaries provide expert vision into current gaps and trends in each area and, hopefully, also provide inspiration for future research.

Alon Eliakim, Expert's Choice Section Editor; Meir Medical Center, Tel-Aviv University Bareket Falk, PES Editor-in-Chief; Brock University 


\title{
Aerobic Exercise and Training During Youth
}

\author{
Expert: Neil Armstrong, University of Exeter
}

This year I have chosen 2 systematic reviews, not because there were no eligible empirical papers but because, collectively, the reviews address what are currently the 2 most popular areas of research in pediatric aerobic fitness and training. The reviews outline what we know, identify serious gaps in our knowledge, and indicate promising avenues of future research. The order of presentation does not reflect preference or priority. My first choice (107) reflects current research programs and focuses on pediatric aerobic (or cardiorespiratory) fitness in relation to current and future health. My second choice (64) focuses on exercise training and is a very welcome systematic review and meta-analysis of the effects of concurrent endurance and strength training on cardiorespiratory fitness (CRF).

Expert Choice 1: Mintjens S, Menting MD, Daams JG, van Poppel MNM, Roseboom TJ, Gemke RJBJ. Cardiorespiratory fitness in childhood and adolescence affects future cardiovascular risk factors: A systematic review of longitudinal studies. Sports Med. 2018;48 (11):2577-2605. doi: 10.1007/s40279-018-0974-5.

Background Although cardiorespiratory fitness (CRF) in childhood and adolescence may be linked to future cardiovascular health, there is currently limited evidence for a longitudinal association. Objectives To provide a systematic review on the prospective association between $\mathrm{CRF}$ in childhood and adolescence and cardiovascular disease (CVD) risk factors at least 2 years later. Methods Using a systematic search of Medline, Embase, and SPORTDiscus, relevant articles were identified by the following criteria: generally healthy children and adolescents between 3 and 18 years of age with CRF assessed at baseline, and a follow-up period of $\geq 2$ years. The outcome measures were CVD risk factors. We appraised quality of the included articles with STROBE and QUIPS checklists. Results After screening 7524 titles and abstracts, we included 38 articles, assessing 44,169 children and adolescents followed up for a median of 6 years. Eleven articles were of high quality. There was considerable heterogeneity in methodology, measurement of CRF, and outcomes, which hampered meta-analysis. In approximately half of the included articles higher CRF in childhood and adolescence was associated with lower body mass index (BMI), waist circumference, body fatness and lower prevalence of metabolic syndrome in later life. No associations between CRF in childhood and adolescence and future waist-to-hip ratio, blood pressure, lipid profile, and glucose homeostasis were observed. Conclusion Although about half of the included articles reported inverse associations between CRF in childhood and adolescence and future BMI, body fatness, and metabolic syndrome, evidence for other CVD risk factors was unconvincing. Many articles did not account for important confounding factors such as adiposity. Recommendations for future research include standardizing the measurement of $\mathrm{CRF}$, i.e. by reporting $V_{2} \mathrm{O}_{2 \max }$, using standardized outcome assessments, and performing individual patient data meta-analyses.

\section{Commentary}

Cardiorespiratory fitness defines the ability to deliver oxygen to the muscles and to utilize it to generate energy to support muscle activity during exercise. Peak oxygen uptake (peak $\mathrm{VO}_{2}$ ) is internationally recognized as the best single measure of youth $\mathrm{CRF}$, and methods of assessing and interpreting youth peak $\mathrm{VO}_{2}$ have been developed over 80 years of laboratory testing (57). Falk and Dotan (56) have presented an excellent 2018 review of the topic but, as in 2017, the year 2018 witnessed a plethora of publications based not on rigorous assessment of peak $\dot{\mathrm{VO}}_{2}$ but on its estimation from field tests. Numerous publications have focused on either the development of variants of the 20-m shuttle run test $(20 \mathrm{mSRT})$ to estimate peak $\mathrm{VO}_{2}(\mathrm{eg}, 13,29,106)$ or the establishment of reference values for cardiometabolic risk in childhood based on peak $\dot{\mathrm{VO}}_{2}$ expressed in ratio with body mass (eg, 5, 26, 152). The 20mSRT has been proposed as a suitable and feasible health indicator for international surveillance in children and adolescents (90) and as a means of evaluating physical activity interventions (89). Others have argued that estimating/ predicting peak $\mathrm{V}_{2}$ from $20 \mathrm{mSRT}$ performance scores is untenable (10), the use of per-body mass ratio scaling is fallacious $(10,168)$, the establishment of thresholds for cardiometabolic risk in children using current criteria is indefensible $(9,10)$, and the use of field test performance estimates of peak $\dot{\mathrm{VO}}_{2}$ to evaluate physical activity interventions is specious (12). In this lively debate, Mintjens and colleagues' (107) thoughtful systematic review of longitudinal studies of the influence of CRF in childhood and adolescence on future cardiovascular risk factors is timely.
The review concluded that higher CRF in children and adolescents was associated with lower body fatness, lower waist circumference, lower body mass index, and a lower prevalence of metabolic syndrome later in life. There was, however, no compelling evidence of an association between CRF during childhood and adolescence and future waist-hip-ratio, blood pressure, lipid profile, or glucose homeostasis. The authors resolved that, "the overall longitudinal association between CRF and CVD risk factors is probably weak to moderate" (107, p. 2600). They noted, however, that there was considerable heterogeneity in methodology and measurement of CRF and recognized that their findings could be "hampered" by confounders that were not uniformly accounted for, such as adiposity at baseline and/or follow-up. They emphasized that adiposity, which does not influence CRF, markedly impacts on performance in field tests purporting to measure CRF (eg, 20mSRT) and confounds associations between estimated CRF and cardiovascular risk factors.

The authors acknowledge at the onset of the review that, "The gold standard to test CRF is by exercising until voluntary exhaustion with direct measurement of the maximum volume of oxygen consumption" (107, p. 2578). Twenty one of the 38 papers included in the review reported measured or estimated peak $\mathrm{VO}_{2}$ but only 11 papers reported objectively measured CRF by using a maximal test with direct assessment of peak $\mathrm{VO}_{2}$. Unfortunately, the majority of papers that directly determined peak $\dot{\mathrm{VO}}_{2}$ only reported it in ratio with body mass. It has been demonstrated theoretically $(169,170)$ and empirically $(9,168)$ that ratio scaling has neither a sound scientific rationale nor statistical justification. Moreover it is age- and maturity-status-driven fat free mass, not body mass, which is the most powerful morphological influence on peak $\mathrm{VO}_{2}(9)$. 
Over 70 years ago Tanner (154) explained how spurious relationships arise when ratio-scaled data are correlated with other health-related variables and how children identified as "at risk" using this technique were likely suffering from "no more formidable a disease than statistical artefact" (154, p. 3). The present review highlights several cases of "statistical artefact". For example (and unsurprisingly), higher peak $\mathrm{VO}_{2}$ in ratio with body mass (ie, in $\mathrm{mL} \cdot \mathrm{kg}^{-1} \cdot \mathrm{min}^{-1}$ ) was associated with lower body fatness but there was no relationship between the 2 variables when peak $\mathrm{V}_{2}$ was expressed in absolute terms (ie, in $\mathrm{L} \cdot \mathrm{min}^{-1}$ ) without reference to body mass. Similarly, a higher peak $\dot{\mathrm{VO}}_{2}$ appeared to be associated with a lower total cholesterol: highdensity lipoprotein ratio; but, once again (as expected), the association was only present when peak $\dot{\mathrm{VO}}_{2}$ was expressed in ratio with body mass.

The review illustrates nicely the essential requirement for all measures to be founded on rigorous scientific methods of assessment when exploring CRF and potential associations with cardiovascular risk factors. The take-home message is clearly that research into the CRF of children and adolescents requires researchers to: (a) precisely define and rigorously assess CRF, (b) appropriately control for the influence of sex-specific concurrent changes in age- and maturity-status-driven morphological and physiological variables when interpreting CRF, and (c) avoid spurious relationships by not using peak $\mathrm{VO}_{2}$ ratio-scaled with body mass in statistical analyses with other health-related variables.

Expert Choice 2: Gabler M, Prieske O, Hortobagyi T, Granacher U. The effects of concurrent strength and endurance training on physical fitness and athletic performance in youth: A systematic review and meta-analysis. Front Physiol. 2018;9(1057):1-13. doi: 10.3389/fphys. 2018.01057.

\begin{abstract}
Combining training of muscle strength and cardiorespiratory fitness within a training cycle could increase athletic performance more than single-mode training. However, the physiological effects produced by each training modality could also interfere with each other, improving athletic performance less than single-mode training. Because anthropometric, physiological, and biomechanical differences between young and adult athletes can affect the responses to exercise training, young athletes might respond differently to concurrent training (CT) compared with adults. Thus, the aim of the present systematic review with metaanalysis was to determine the effects of concurrent strength and endurance training on selected physical fitness components and athletic performance in youth. A systematic literature search of PubMed and Web of Science identified 886 records. The studies included in the analyses examined children (girls age 6-11 years, boys age 6-13 years) or adolescents (girls age 12-18 years, boys age 14-18 years), compared CT with single-mode endurance (ET) or strength training (ST), and reported at least one strength/power-(e.g., jump height), endurance-(e.g., peak $\mathrm{VO}_{2}$, exercise economy), or performance-related (e.g., time trial) outcome. We calculated weighted standardized mean differences (SMDs). CT compared to ET produced small effects in favor of CT on athletic performance $(n=11$ studies, $\mathrm{SMD}=0.41, p=0.04)$ and trivial effects on cardiorespiratory endurance $(n=4$ studies, $\mathrm{SMD}=0.04, p=0.86)$ and exercise economy $(n=5$ studies, $\mathrm{SMD}=0.16, p=0.49)$ in young athletes. A sub-analysis of chronological age revealed a trend toward larger effects of CT vs. ET on athletic performance in adolescents $(\mathrm{SMD}=0.52)$ compared with children $(\mathrm{SMD}=0.17)$. CT compared with ST had small effects in favor of CT on muscle power $(n=4$ studies, $\mathrm{SMD}=0.23, p=0.04)$. In conclusion, CT is more effective than single-mode ET or ST in improving selected measures of physical fitness and athletic performance in youth. Specifically, CT compared with ET improved athletic performance in children and particularly adolescents. Finally, CT was more effective than ST in improving muscle power in youth.
\end{abstract}

\section{Commentary}

The trainability of pediatric aerobic fitness is shrouded in controversy, with the concept of a "maturation threshold" or "trigger point" below which the effects of training will be minimal or will not occur embedded in the pediatric literature. There is, however, no compelling empirical evidence to support the hypothesis. Data from "high-quality" studies clearly show that percentage gains in peak $\dot{\mathrm{V}}_{2}$ following training of the appropriate frequency, duration, and intensity during childhood and adolescence are independent of age, maturity status, and sex $(8,103,122)$. Knowledge of the physiological mechanisms underlying gains in peak $\dot{\mathrm{VO}}_{2}$ with training and whether they are influenced by age, sex, and maturity status is, however, less clear, and founded largely on responses to continuous intensity exercise training (CIET) programs. Changes in peak $\mathrm{V}_{2}$ induced by CIET programs have been primarily attributed to enhanced oxygen delivery to the muscles through increased stroke volume, but whether this is due to morphological and/or functional adaptations and how they are influenced by sex, age, and maturity status remains unclear (11).

The origins of high-intensity interval training (HIIT) with adult athletes can be traced back at least to the beginning of the 20th century. Surprisingly, in the light of young people being able to recover more rapidly from repeated bouts of high-intensity exercise than adults (130), it is only recently that a concerted research effort has focused on HIIT with youth. Recent systematic reviews have reported that HIIT is an effective and time-efficient means of enhancing youth peak $\dot{\mathrm{VO}}_{2}$ in both childhood and adolescence, but these conclusions are based on only 8 to 13 published investigations which met the eligibility criteria of the reviews $(35,49,157)$. No "high-quality" studies appear to have investigated the mechanisms underpinning HIIT-induced changes in youth peak $\dot{\mathrm{VO}}_{2}$ but HIIT interventions with adults have suggested that enhanced oxygen utilization by the muscles rather than oxygen delivery to the muscles is primarily responsible for increased peak $\mathrm{VO}_{2}$ (68). Extrapolation of adult data to children and adolescents must be done with extreme caution, but the intriguing question which arises is as follows: If CIET stimulates enhanced oxygen delivery and HIIT induces increased oxygen utilization, would an interplay between the 2 protocols optimize increases in youth peak $\dot{\mathrm{VO}}_{2}$ ? (11).

Gabler and colleagues' (64) innovative contribution introduces another factor into this intriguing mix. The authors systematically compare the effects of concurrent strength and endurance (ie, aerobic) training (CT) programs with single-mode strength (ST) or endurance (ET) training programs. The review is the latest of a series of excellent systematic reviews and meta-analyses (e.g. 71, 95, 147) that have emerged from the KINGS Study, which has made significant contributions to our understanding of strength training in the long-term development of youth athletes. In addition 
to a ST element, the present review also addresses whether CT increases peak $\dot{\mathrm{VO}}_{2}$, and/or exercise economy, and/or athletic performance in youth athletes more than single-mode ET. As young people move through childhood and adolescence they experience, with individual timing and tempo, concurrent changes in morphological and physiological variables. They recalibrate the interactions between their changing bodies and environmental constraints and modify the development of motor skills to enhance a variety of activity- and sport-related motor performances. It is noteworthy therefore that Gabler and colleagues (64) recognize that, in the context of the development of youth athletes, a high peak $\dot{\mathrm{VO}}_{2}$ may be a prerequisite of elite performance in some sports but that success in many sports relies as much (or more) on sportrelated motor skills and/or the ability to economically sustain performance at various levels of submaximal exercise. Exercise economy is an important component of performance and many world-class athletes have benefited through training-induced increases in their exercise economy without concomitant changes in peak $\dot{\mathrm{VO}}_{2}(11)$.

Gabler and colleagues (64) noted that 4 studies reported CT and ET effects on peak $\mathrm{VO}_{2}$ and 5 studies reported CT and ET effects on exercise economy expressed as $\mathrm{VO}_{2}$ at a submaximal intensity during swimming, running, skiing, or rowing. On the basis of weighted standardized mean differences it was concluded that, compared to single-mode ET, CT had a trivial effect in adolescent athletes. In these 9 studies plus 2 others, a measure of athletic performance was measured as the time of, or the mean velocity during, a time trial. A moderate effect of CT over ET was reported in adolescent athletes with a trivial effect in child athletes. The authors acknowledge that their conclusions are constrained by a paucity of eligible studies, small sample sizes, and, in some cases, uncontrolled training volumes. Yet, they recommend that both ST and ET should be included in training programs to increase endurance performance in youth athletes and to improve physical fitness in nonathletic youth.

Gabler et al. (64) highlight the availability of only "a handful of studies", the heterogeneity of the data, and the limited rigorous research which has been devoted to exploring the effects of both muscle strength and CRF training within a training cycle. The review suggests several interesting lines of enquiry into the trainability of children and adolescents and the ideal approach for optimizing the long-term development of youth athletes. Research questions raised include: (a) Does CT potentiate the individual effects produced by ET and ST more than ET and ST alone? (b) Do ET and ST "interfere" with each other and produce inferior gains in ET and ST? (c) Does the effectiveness of CT with youth athletes depend on the type of activity or sport?

Throughout their review the authors rightly emphasize the importance of considering sex and biological age when interpreting training adaptations. They indicate that, on balance, a ST before ET sequence "may produce the best results in adolescent athletes but the order does not seem to differentially affect training adaptations in children" $(64$, p. 11). The authors acknowledge that this view is based on limited empirical evidence and clearly more research on this issue is needed, perhaps with fine tuning of the global definition of "adolescents" in relation to sex, chronological age, and maturity status.

The burning issue in pediatric aerobic trainability remains that causal mechanisms of CIET-, HIIT-, and CT-induced changes in $\mathrm{CRF}$ and their modulation by sex, age, and maturity status remain to be resolved. The application of noninvasive technologies, such as near-infrared spectroscopy, magnetic resonance imaging and spectroscopy, Doppler echocardiography, and thoracic bioimpedance, to rigorously-designed, well-controlled, and varied training interventions with appropriate evaluations could provide further insights and open new lines of research.

\title{
Neuromuscular Physiology, Exercise, and Training During Youth
}

\author{
Expert: David G. Behm, Memorial University of Newfoundland
}

Expert Choice 1: Moran J, Sandercock G, Ramirez-Campillo R, Clark CCT, Fernandes JFT, Drury B. A meta-analysis of resistance training in female youth: Its effect on muscular strength, and shortcomings in the literature. Sports Med. 2018;48(7):1661-1671. doi: 10. 1007/s40279-018-0914-4

\begin{abstract}
Background Resistance training is an effective way to enhance strength in female youth but, to date, no researcher has metaanalysed its effect on muscular strength in that population. Objectives This meta-analysis characterised female youths' adaptability to resistance training (RT). A second objective was to highlight the limitations of the body of literature with a view to informing future research. Data Sources Google Scholar, PubMed, Web of Science. Study Eligibility Criteria Resistance training interventions in healthy females with a mean age between 8 and 18 years. Programmes of between 4 and 16 weeks' duration that included a control group. Study Appraisal and Synthesis Methods The inverse-variance random effects model for meta-analyses was used because it allocates a proportionate weight to trials based on the size of their individual standard errors and facilitates analysis whilst accounting for heterogeneity across studies. Effect sizes, calculated from a measure of muscular strength, are represented by the standardised mean difference and are presented alongside $95 \%$ confidence intervals. Results The magnitude of the main effect was 'small' $(0.54,95 \%$ confidence interval: $0.23-0.85)$. Effect sizes were larger in older $(>15$ years; $\mathrm{ES}=0.72[0.23-1.21]$ vs. $0.38[-0.02-0.79])$, taller $(>163 \mathrm{~cm} ; \mathrm{ES}=0.67[0.20-1.13]$ vs. $0.55[0.08-1.02])$ and heavier $(<54 \mathrm{~kg}$; $\mathrm{ES}=0.67$ [0.30-1.03] vs. $0.53[-0.00-1.06])$ participants. Conclusions and Implications of Key Findings Resistance training is effective in female youth. These findings can be used to inform the prescription of RT in female youth.
\end{abstract}

Expert Choice 2: Moran J, Clark CCT, Ramirez-Campillo R, Davies MJ, Drury B. (2018). A meta-analysis of plyometric training in female youth: Its efficacy and shortcomings in the literature. J Strength Cond Res. 2018; Epub ahead of print. doi: 10.1519/JSC. 0000000000002768

This meta-analysis characterized female youths' adaptability to plyometric training (PT). A second objective was to highlight the limitations of the body of literature with a view to informing future research. Fourteen studies were included in the final analysis. 
The effect size (ES $=$ Hedges' $g$ ) for the main effect of vertical jump performance was "small" (ES $=0.57,95 \%$ confidence interval: $0.21-0.93)$. Effect sizes were larger in younger ( $<15$ years; $\mathrm{ES}=0.78[0.25-1.30]$ vs. $0.31[-0.18$ to 0.80$])$, shorter $(<163 \mathrm{~cm} ; \mathrm{ES}=$ 1.03 [0.38-1.68] vs. 0.25 [20.20 to 0.70]), and lighter $(<54 \mathrm{~kg} ; \mathrm{ES}=1.14$ [0.39-1.89] vs. 0.26 [ -0.15 to 0.67$])$ participants. Programming variables seemed to influence adaptive responses with larger effects in interventions which were longer ( 8 weeks; $\mathrm{ES}=1.04[0.35-1.72]$ vs. 0.24 [ -0.11 to 0.59$]$ ), had greater weekly training frequency $(>2 ; \mathrm{ES}=1.22[0.18-2.25]$ vs. $0.37[0.02-$ $0.71]$ ), and whose sessions were of longer duration ( $\geq 30$ minutes $E S=1.16$ [0.14-2.17] vs. 0.33 [0.03- 0.63]). More than 16 sessions per program $(0.85$ [0.18- 1.51$])$ was more effective than exactly 16 sessions $(0.46$ [0.08-0.84]) which, in turn, was more effective than less than 16 (0.37 [ -0.44 to 1.17]). These findings can inform the prescription of PT in female youth.

Expert Choice 3: Sommi C, Gill F, Trojan JD, Mulcahey MK. Strength and conditioning in adolescent female athletes. Phys Sportsmed. 2018;46(4):420-426. doi: 10.1080/00913847.2018.1486677

\begin{abstract}
Despite evidence that strength and conditioning $(\mathrm{S} \& \mathrm{C})$ programs decrease injury risk and increase sport performance, young females are rarely offered S\&C programs comparable to those of their male counterparts. The purpose of this study was to evaluate the current body of available literature regarding $\mathrm{S} \& \mathrm{C}$ in adolescent female athletes, describe potential benefits, and generate recommendations for $\mathrm{S} \& \mathrm{C}$ programs for female adolescent athletes. This systematic review was performed in accordance with the Preferred Reporting Items for Systematic Reviews and Meta-Analyses guidelines. Systematic searches of the PubMed and Google Scholar databases were conducted using the following keywords: 'female athletes,' 'injury prevention,' 'adolescent,' 'physical fitness,' 'strength,' 'female adolescent,' and 'conditioning.' Studies included in this review evaluated the effectiveness of S\&C protocols and/or the habits and attitudes of coaches and athletic trainers working with female adolescent athletes. Seven articles evaluating S\&C programs for the adolescent female athlete were used as the basis for this systematic review. These articles described current protocols and/or factors that should be taken into account when designing S\&C programs. The identified articles focused on improving the strength of adolescent female athletes, decreasing the risk of injury, and exposing female athletes to the benefits of $\mathrm{S} \& \mathrm{C}$ that are routinely afforded to their male counterparts. Despite the critical potential benefits of $S \& C$ training, such as improved landing mechanics, coaches and athletic trainers do not routinely implement S\&C programs for female adolescent athletes. The lack of such programs is largely due to misconceptions surrounding female athletes, such as the perception that females fear bulking up. S\&C programs for adolescent female athletes should incorporate stretching of the hip adductors, targeted hamstring, gluteal and quadriceps strengthening, and a synergistic adaptation model, which tailors training protocols to an athlete's pubertal stage.
\end{abstract}

\section{Commentary}

It is of ubiquitous bias, whether intended or unintended, in the neuromuscular exercise physiology literature that investigations of male participants constitute a much higher predominance than the examination and analysis of female responses $(108,109,151)$. It has come to light in the health sciences and medical literature that such a bias can have dire consequences when health practitioners, such as doctors, are not cognizant of female responses, symptoms, and reactions that are distinct from men. For example, men and women can differ in their symptoms or responses to heart attacks (myocardial infarctions), strokes, and autoimmune diseases, as well as diseases involving gastroenterology, hepatology, nephrology, endocrinology, haematology, and neurology, thus influencing sexspecific differences in treatment and pharmacology (131). There are both sex (physiological) and gender (sociological) differences that can affect medical treatment. Sex factors can include differences in reproductive function, concentrations of sexual hormones, gene expression, and the higher body fat composition in women. By contrast, gender is associated with behavior, lifestyle, and life experience. These differences can impact access and use of health care as well as the behavioral attitudes of medical personnel (131). Whereas there are more than 10,000 articles examining sex and gender differences in the medical field literature, the examination of female neuromuscular responses to exercise and training (sport and exercise science) is not as well established.

In 2018, 2 meta-analyses examining sex differences with resistance (109) and plyometric (108) training and the literature's shortcomings were published. In addition, another review on strength and conditioning in adolescent female athletes was published by Sommi et al (151). Hopefully, these reviews serve as beacons to highlight the need for further research in neuromuscular exercise physiology and training of female responses and adaptations. In comparison to the vast literature on male training responses, the two Moran et al meta-analyses identified only 11 and 14 relevant studies investigating resistance and plyometric training responses, respectively. Similarly, Sommi et al (151) wished to evaluate and generate recommendations for strength and conditioning in adolescent female athletes, but could only find 7 relevant articles.

The more scant body of female youth literature prevented Moran et al in either meta-analysis (resistance or plyometric training reviews) from examining or identifying maturational (ie, pre-, peak-, and postpeak height velocity) differences, which have been classified extensively with the more abundant young male literature. Maturation status is an essential factor differentiating performance levels in young males but is less pervasive in the young female literature. As Moran and colleagues lament, "Given that RT [resistance training] can reduce injuries in females (96) and, also, that greater levels of strength are thought to prevent injury $(14,54)$, it is curious that interventions which directly address the impact of RT on a measure of muscular strength are so scarce in female youth. .." (109, p. 1667).

A common problem in the training literature is the pooling of performance data of both females and males for analysis within the same studies. Such a practice provides a hybrid response that nullifies and is independent of any population-specific effects $(108,109)$. For example, at the most basic level, there are innumerable studies with both male and female participants that provide mean age and anthropometric statistics for the group as a whole. How is it possible to replicate such a study when the anthropometric characteristics represent some hybrid male-female subject and thus do not delineate the actual mean characteristics (or responses) of each sex? 
The Moran et al $(108,109)$ meta-analyses reported that mean resistance and plyometric training responses were of a smaller magnitude than those observed in boys. With plyometric training, older, taller, and heavier female participants show substantially smaller effects than younger, smaller, and lighter counterparts. They rationalized that, during peak height velocity, while males gain more absolute muscle mass, girls obtain less muscle mass with greater fat mass, which would negatively impact their relative strength and thus ability to jump or bound higher. Younger girls with less fat and muscle mass more similar to young boys would facilitate better responses to plyometric training.

In contrast to plyometric training, resistance training was more effective (moderate vs small effects) in older, taller, and heavier girls. Whereas younger, leaner girls respond better to plyometric training than older, heavier girls, evidence seems to indicate that more biologically-mature female youth may have greater magnitude resistance training adaptations. Similar to boys, resistance training responses are greater during and after peak height velocity, although the reporting of peak height velocity stages are scarce in female studies. For both sexes, this might be partially attributed to maturation-related increases in muscle mass as well as the potentially negative effects of early puberty, such as impaired sensorimotor function (126). Furthermore, Moran and colleagues' meta-analysis elucidates that a number of resistance training studies do not include measures of absolute strength but rather measure relative strength, such as with the vertical jump, which may mask the true training adaptation due to the disadvantage of girls' higher fat mass despite enhanced absolute strength.

The Sommi et al (151) review emphasized many of the physiological hurdles that girls may experience during puberty, such as increases in body mass and height and the onset of menstruation, that could affect sports participation and lead to emotional stress. Female-specific factors place girls at higher risk for musculoskeletal injuries, such as stress fractures and anterior cruciate ligament tears. These factors can include anatomical differences, altered biomechanics, and hormonal changes. Girls may also experience lower quadriceps and hip adduction strength and are susceptible to the female athlete triad (poor energy availability, irregular menses, and low bone density). Thus, Sommi et al (151) recommend "synergistic training adaptations" (52). This type of training method suggests that strength and conditioning programs should complement the physiological adaptations occurring throughout childhood and adolescence. While strength and conditioning training may help minimize the female risk of sustaining these injuries, physical education teachers and coaches of girls were less likely to be as experienced or appropriately certified for implementing strength and conditioning programs than coaches for boys. Hence, the likelihood of a girl receiving an appropriate, task-specific strength training program commensurate with her maturational level and taking into account sex differences is much less likely than for a boy.

In conclusion, the Moran et al meta-analyses $(108,109)$ and the Sommi et al review (151) highlight how girls can benefit similarly to boys from neuromuscular resistance training programs. However, while they highlight specific sex-related differences and challenges in the girls' training responses, the female literature is still too limited to provide the comprehensive neuromuscular training evaluations that are available for boys. Hopefully these reviews spark greater interest in recruiting and investigating girls' neuromuscular training responses and adaptations.

\title{
Endocrinology and Exercise in Youth
}

\author{
Expert: Alon Eliakim, Meir Medical Center, Tel-Aviv University
}

Expert Choice 1: Cadegiani FA, Kater CE. Hormonal response to non-exercise stress test in athletes with overtraining syndrome: Results from the Endocrine and metabolic Responses on Overtraining Syndrome (EROS) - EROS-STRESS. J Sci Med Sport. 2018;21(7):648653. doi: $10.1016 /$ j.jsams.2017.10.033

\begin{abstract}
Objectives: Overtraining syndrome (OTS) leads to worsened sports performance and fatigue. The pathophysiology of OTS has not been entirely elucidated, and there is a lack of accurate markers for its diagnosis. Changes in hormonal responses implicated in OTS were stimulated by exercise, which has limited their interpretation. Hence, we aimed to evaluate growth hormone (GH) and prolactin responses to a gold-standard and exercise-independent stimulation test, the insulin tolerance test (ITT). Design: Volunteers were recruited and divided into OTS-affected athletes (OTS), healthy athletes (ATL), and healthy non-active subjects (NCS) groups, after general and specific inclusion and exclusion criteria. Methods: We evaluated the responses of growth hormone $(\mathrm{GH})$ and prolactin to the ITT, and compared between groups. Results: A total of 51 subjects were included (OTS, $\mathrm{n}=$ 14 , ATL, $n=25$, and NCS, $n=12)$. OTS disclosed significantly lower basal levels of GH $(p=0.003)$ and prolactin $(p=0.048)$, and GH $(\mathrm{p}=0.001)$ and prolactin $(\mathrm{p}<0.001)$ responses to ITT $(\mathrm{p}=0.001)$, compared to ATL, but similar to NCS. OTS showed a later rise in GH levels in response to hypoglycemia, compared to ATL, but not to NCS. We suggest cutoffs for GH and prolactin levels to aid in the diagnosis of OTS. Conclusions: OTS-affected athletes show reduced GH and prolactin basal levels and responses to a non-exercise stress test compared to healthy athletes, but not to sedentary subjects.
\end{abstract}

\section{Commentary}

Overtraining syndrome is characterized by intense fatigue and reduced sport-specific performance due to an imbalance between the training load and the ability of the athlete to tolerate it (93). While there are no objective definite, accurate, and diagnostic markers of overtraining syndrome, changes in several hormonal responses to exercise have been suggested $(27,104)$. However, since these hormonal changes were studied mainly in response to exercise, they may result from differences in physical conditioning and the reduced exercise performance in overtraining and not from hormonal dysfunction per se. Thus the aim of the Cadegiani and Kater study (28) was to assess the hormonal response (ie, growth hormone-GH and prolactin) to a nonexercise, insulin-tolerance test (ITT) in adult male athletes.

The hormonal response to exercise was compared between healthy athletes, athletes with overtraining syndrome, and sedentary controls. The study demonstrated several very important findings. First, the $\mathrm{GH}$ and prolactin responses to ITT were significantly greater among the healthy athletes compared to 
controls. This suggests proper training adaptation, and, in the case of elevated $\mathrm{GH}$, it may mediate the anabolic adaptation necessary to training-induced muscle hypertrophy. Whether the response of these hormones can serve as an additional tool to assess optimal training needs further research. Second, and perhaps the most striking finding of the study, GH and prolactin response to ITT in overtrained athletes was blunted compared to the healthy athletes, suggesting that this hormonal maladaptation is more generalized and not related only to exercise. This also suggests that overtraining is characterized by a possible "deconditioning process" of the hormonal benefits acquired by optimal training. Moreover, the authors were able to provide useful cutoffs for diagnosis of overtraining (eg, $\mathrm{GH}$ response of $<5 \mathrm{mic} / \mathrm{L}$ has $77 \%$ accuracy as a criterion for overtraining whereas a response of $>14 \mathrm{mic} / \mathrm{L}$ has $100 \%$ negative predictive value for overtraining). The main question that was not answered by the present study was whether this blunted hormonal response can be seen prior to the overtraining-related reduced competitive performance and can be used as an early diagnostic and prevention sign. Finally, the athletes with overtraining remained asymptomatic during mild to moderate episodes of hypoglycemia that occurred during the ITT. The authors speculated that similar to insulin-dependent diabetic patients, this is likely due to repeated unnoticed episodes of hypoglycemia during training leading to reduced adrenergic sensitization and manifestations (175). Therefore, the authors recommended that athletes with suspected overtraining must increase carbohydrate intake prior to, during, and after training in order to prevent hypoglycemic episodes.

Future studies are needed to evaluate whether these results are also applicable for adolescent and female athletes, and for overtraining in different sport types (eg, individual vs team, land vs water, contact vs noncontact, etc). In particular, the possible effect of reduced $\mathrm{GH}$ secretion during adolescence, a time of rapid linear growth, should be evaluated.

Expert Choice 2: Abderrahman AB, Rhibi F, Ouerghi N, Hackney AC, Saeidi A, Zouhal H. Effects of recovery mode during high intensity interval training on glucoregulatory hormones and glucose metabolism in response to maximal exercise. J Athl Enhanc 2018;7 (3):292. doi: $10.4172 / 2324-9080.1000292$

Catecholamines [adrenaline (A) and noradrenaline (NA)] are known to stimulate glucose metabolism at rest and in response to maximal exercise. However, training and recovery mode can alter theses hormones. Thus our study aims to examine the effects of recovery mode during High-intensity Interval Training (HIIT) on glucoregulatory hormone responses to maximal exercise in young adults. Twenty-four male enrolled in this randomized study, assigned to: control group $(\mathrm{cg}, \mathrm{n}=6)$, and two HIIT groups: intermittent exercise (30 s run/30 s recovery) with active $(\arg , \mathrm{n}=9)$ or passive $(\mathrm{prg}, \mathrm{n}=9)$ recovery. arg and prg performed HIIT 3 times weekly for 7 weeks. Before and after HIIT, participants undergo a Maximal Graded Test (MGT). Plasma catecholamines, glucose, insulin, growth hormone (Gh) and cortisol were determined at rest, at the end of MGT, after 10 and 30 min of recovery. After training V02max and Maximal Aerobic Velocity (MAV) increased significantly $(\mathrm{p}<0.05)$ in arg. After HIIT and in response to MGT plasma glucose increase significantly $(\mathrm{p}=0.008)$ lesser in arg compared to prg whereas insulin concentrations were similar. The glucose/ insulin ratio was significantly lower at MGT end $(\mathrm{p}=0.033)$ only in arg after training. After HIIT, in response to MGT, plasma A, NA, cortisol and Gh concentrations were significantly higher only in arg ( $\mathrm{p}<0.05)$. HIIT using active recovery is beneficial for aerobic fitness, plasma glucose and glucoregulatory hormones better than HIIT with passive recovery. These findings suggest that HIIT with active recovery may improve some metabolic and hormonal parameters in young adults

\section{Commentary}

The use of additional supporting aids for the training process plays only a relatively minor role in improving competitive athletic performance. However, in competitive sports, where one hundredth of a second or a few millimeters could make a difference between fame and shame in the life of an athlete, the search for legal methods to improve training ability and performance becomes critical.

The development of methods to enhance the recovery of elite athletes from intense training and/or competition has been a major target of both the athletes and their accompanying staff for many years. The performance advantage of active recovery during repeated high-intensity, short-term exercise is well established (6). It is suggested that active recovery promotes blood flow to the joints and muscles, reduces the accumulation of lactic acid in the muscles, minimizes postexercise stiffness and discomfort, counteracts inflammation, and alleviates fatigue (44). However, other studies demonstrated that passive recovery was better than active recovery for repeated dribbling sprint test performance and for subjective fatigue estimation in young soccer players (67). In contrast to the many studies on the effect of recovery modes on lactate clearance, fatigue, and perceived exertion, the effect of recovery on the hormonal and anabolic effects of exercise training is much less studied.
Abderrahman et al (4) investigated the effect of active versus passive recovery during high-intensity interval training (HIIT) on glucoregulatory hormone response to maximal exercise tests in 21-year-old male physical education students. Their main finding was that, along with a greater improvement in aerobic fitness, the $\mathrm{GH}$ response to a maximal graded exercise test was greater following HIIT with active recovery compared to HIIT with passive recovery. This suggests that, along with training-associated improvements of fitness measures such as power and anaerobic and aerobic characteristics, part of the adaptation to training is that a single practice becomes more anabolic. The unique finding of Abderrahman et al (4) was that this anabolic adaptation was influenced primarily by the recovery modality and not by the physical exertion itself. Along with that, we previously demonstrated (113) that local cold-packs application, another common recovery modality, during the recovery from a $4 \times 250-\mathrm{m}$ interval training session was associated with significant blunting of the increase of the anabolic factors IGF-I and IGF binding protein-3. This raises an important, relatively new concept that different recovery modalities are important not only for the recovery from the previous practice and for better preparation for the next training session, but may also blunt or augment the anabolic effect of training itself. Further studies are needed to explore the beneficial use of anabolic, catabolic, and inflammatory marker measurement in many other aspects of recovery from exercise in male and female adolescent and adult athletes. 


\title{
Youth Resistance Training: Play Now or Pay Later
}

\author{
Expert: Avery D. Faigenbaum, The College of New Jersey
}

Expert Choice 1: Castro-Piñero J, Perez-Bey A, Cuenca-Garcia M, Cabanas-Sanchez V, Gómez-Martínez S, Veiga OL, Marcos A, Ruiz JR, on behalf of the UP\&DOWN Study Group. Muscle fitness cut points for early assessment of cardiovascular risk in children and adolescents. J Pediatr. 2018; Epub ahead of print. doi: 10.1016/j.jpeds.2018.10.026

\begin{abstract}
Objectives To study the cross-sectional and longitudinal (2-year follow-up) association between muscle fitness and cardiovascular disease (CVD) risk factors in youth; whether there are muscle fitness cut points associated with CVD risk (cross-sectional); and whether the health-related muscle fitness cut points identified at baseline are associated with CVD risk 2 years later. Study design In total, 237 children (110 girls) aged 6-10 years and 274 adolescents (131 girls) aged 12-16 years with complete data were included in the study (10.3\% drop out). The handgrip strength and the standing long jump tests were used to assess muscle fitness. CVD risk score was computed with sum of 2 skinfolds, systolic blood pressure, insulin, glucose, triglycerides, and total cholesterol/high density lipoprotein cholesterol. Results Muscle fitness at baseline was associated inversely with single CVD risk factors and CVD risk score at baseline and 2-year follow-up (all $P<.05$ ). Receiver operating characteristics curve analyses showed a significant discriminating accuracy of handgrip strength in identifying CVD risk in children and adolescents (boys: $\geq$ 0.367 and $\geq 0.473$; girls: $\geq 0.306$ and $\geq 0.423 \mathrm{~kg} / \mathrm{kg}$ body mass, respectively, all $P<.001)$. Similarly, the standing long jump cut points for children and adolescents were $\geq 104.5$ and $\geq 140.5$ in boys, and $\geq 81.5$ and $\geq 120.5 \mathrm{~cm}$ in girls, respectively (all $P<.05$ ). These cut points were associated with CVD risk 2 years later (all $P<.01$ ). Conclusions Muscle fitness is associated with present and future cardiovascular health in youth, and is independent of cardiorespiratory fitness. It should be monitored to identify youth at risk who could benefit from intervention programs.
\end{abstract}

\section{Commentary}

Too often, contemporary physical activity guidelines for children focus on aerobic exercise whereas recommendations for muscular fitness seem to be less important. In support of this observation, the 2018 Physical Activity Guidelines for Americans report states that, "Children do not usually do or need formal muscle-strengthening programs, such as lifting weights" (161, p. 49). This view undermines the critical importance of building a strength reserve early in life in order to set the stage for ongoing participation in a variety of physical activities throughout the life course. As noted by others, the concept of reserves refers to a special type of resource that can be used (or not) to overcome adverse life events or protect against the negative effects of aging (36). From this perspective, the lack of a strength reserve can be considered a vulnerability that may impede one's ability to perform physical activities or cope effectively with stressors. Recent attention has focused on the importance of identifying health-related muscular fitness cut points in children and adolescents since cardiorespiratory fitness cut points to avoid cardiovascular disease (CVD) have already been established $(2,137)$. A low level of muscular strength is a well-recognized risk factor for functional disability and mortality in older adults $(66,94,102)$ and reports indicate that weaker youth are also susceptible to adverse health consequences $(17,70,148)$. These findings underscore the importance of identifying and treating pediatric dynapenia as an independent risk factor for physical inactivity and chronic disease (55). By definition, pediatric dynapenia is an acquired condition characterized by low levels of muscular strength and power and consequent functional limitations not caused by neurological or muscular disease (53).

Castro-Piñero and colleagues examined the cross-sectional and longitudinal association between muscle fitness (assessed with the handgrip strength and standing long jump) and CVD risk factors in Spanish children and adolescents over a 2-year period (30). They found that poor levels of muscular fitness in children and adolescents were associated with a higher risk of CVD at baseline and 2 years later. Notably, they identified muscular fitness cut points that were associated with a more favorable cardiovascular health profile. Children and adolescents who met the handgrip strength or standing long jump cut points had low values of CVD risk 2 years later. Since the observed associations between muscular fitness and cardiovascular health were independent of cardiorespiratory fitness, these findings reinforce the importance of muscular fitness as a marker of health in schoolage youth. Others also found that handgrip strength was associated with cardiovascular health in youth from the United States (121), Canada (133), and Colombia (128).

Notwithstanding the value of assessing cardiorespiratory fitness to identify youth at increased risk of disease, health-related fitness testing should also assess muscular strength and power. Indeed, current recommendations call for the inclusion of the handgrip strength and standing long jump tests to measure upper and lower body strength in children and adolescents (80). Given the link between poor muscle strength and adverse health outcomes $(17,70,148)$, it seems prudent to identify and treat weak children early in life before they become resistant to interventions later in life. Muscle strength testing should be part of school-based physical education fitness assessments. Further, health care providers could use these strength tests during well-child visits to screen for muscle weakness and detect other clinically-relevant health concerns. A young child identified as weak should be treated with the same energy and resolve as a child with other CVD risk factors.

The decline and disinterest in physical activity begins early in life and there are no pharmacological treatments for physical inactivity $(141,165)$. School care and health care should focus on the identification and treatment of youth with muscle weakness and should recognize muscle strength as a biomarker of health. At present, modern-day youth are less active, more obese, and weaker than past generations, and it is likely that the economic burden of managing health complications arising from already existing disease will continue $(125,165)$. A take home message is that the handgrip strength or standing long jump has the potential to be used as a simple and inexpensive screening tool to alter the current trajectory toward physical inactivity and related comorbidities. 
Expert Choice 2: Henriksson H, Henriksson P, Tynelius P, Ortega FB. Muscular weakness in adolescence is associated with disability 30 years later: A population-based cohort study of 1.2 million men. Br J Sports Med. 2018; Epub ahead of print. doi: 10.1136/bjsports2017-098723

\begin{abstract}
Objective To investigate the associations of muscular strength in adolescence with later disability pension (DP), across different body mass index (BMI) categories and in combination with aerobic fitness. Method This prospective cohort study consisted of males aged 16-19 years, recruited from the Swedish military conscription register between 1969 and 1994. A total of 1,212,503 adolescents met all the inclusion criteria and were therefore included in the analyses. Knee extension, handgrip and elbow flexion strength and aerobic fitness (bicycle ergometer test) were measured during conscription. Causes of DP were retrieved from the Social Insurance Office between years 1971 and 2012 (average follow-up time: 29.6 years). Results Knee extension strength in adolescence was inversely associated with men's risk of obtaining DP due to all causes (HR 1.40, $95 \%$ CI 1.36 to 1.44 for lowest vs highest strength quintile). Thus, muscular weakness was associated with DP. The risk associated with low muscular strength differed between specific causes of DP and the strongest associations were found for psychiatric, nervous system and other causes (HRs between 1.47 and 1.90 for lowest vs highest quintile). Being strong was associated with lower DP risk across BMI categories and being unfit, weak and obese was associated with the highest DP risk (HR 3.70, 95\% CI 2.99 to 4.58). Conclusion There was a strong association between muscular weakness and disability. A combination of muscular weakness and low aerobic fitness was an especially important risk factor for disability. This adds weight to call for muscular strength and fitness enhancing exercise for adolescents in all BMI categories.
\end{abstract}

\section{Commentary}

The importance of building and maintaining high strength reserves throughout childhood and adolescence should not be overshadowed by generic recommendations to simply increase moderate-to-vigorous physical activity (MVPA). Secular trends in measures of muscular fitness indicate that modern-day youth are weaker than previous generations $(61,138)$ and there is evidence that low levels of muscular strength during the growing years tend to track into adulthood (63). Epidemiological findings indicate that a vast majority of modern-day youth are not meeting current physical activity recommendations that call for at least 60 minutes of MVPA daily (16). A contemporary corollary of these troubling trends is a high rate of cardiometabolic, musculoskeletal, and psychosocial risk factors and diseases that are challenging to manage and costly to individuals and society. The global cost of physical inactivity is estimated to be INT \$54 billion per year in direct health care costs (173).

Traditionally, youth have been encouraged to participate primarily in aerobic activities in order to improve markers of cardiorespiratory health (161). Yet, gains in muscular strength are also powerful determinants of health $(116,148)$. Higher levels of muscular strength in youth are associated with lower rates of adiposity, cardiovascular disease, and metabolic risk factors $(62,148)$. Moreover, stronger adolescents have a lower risk of death from suicide and are less likely to have any psychiatric diagnosis such as schizophrenia or mood disorders (117). To alter the current trajectory toward physical inactivity and related comorbidities, research is needed to study the link between muscle weakness and longitudinal outcomes in order to increase the effectiveness of targeted interventions aimed at increasing muscle strength.

Henriksson and colleagues expanded our understanding of the influence of muscular weakness in adolescence on markers of disability later in life (76). They followed over 1.2 million male adolescents from the Swedish military between 1969 to 1994. Baseline strength measures included knee extension, elbow flexion, and handgrip strength. A diagnosis of a disability was confirmed by a physician and included individuals whose working capacity was chronically reduced by at least $25 \%$ due to illness or injury. During the study period, $5.7 \%$ of the participants (mean age $42.2 \pm 8.4$ years) were granted disability due to a medical condition. They found low levels of muscular strength during adolescence were associated with a higher risk of disability and the association was strongest for knee extension strength followed by handgrip strength. While a combination of muscular weakness and low aerobic fitness was related to a higher risk of disability than either condition alone, being strong and aerobically fit attenuated the risk of disability in both the normal weight and obese groups. Interestingly, psychiatric disorders were the most frequent cause of disability in this investigation.

As the authors indicate, disability places a great burden on the individual and on society and therefore concerted efforts are needed to enhance muscular strength and fitness in all body mass index categories throughout childhood and adolescence. From a public health perspective, there is a critical need during childhood and adolescence to increase muscular strength and build strength reserves in order to develop healthy, resilient, and capable youth who are prepared for ongoing participation in active play and sport activities. Since prerequisite levels of muscular strength beget participation in MVPA, youth with poor muscular strength may not be able to break through a strength barrier that would allow them to catch up to their stronger peers and participate regularly in MVPA. Consequently, it is likely that the gap between weaker and stronger youth will widen over developmental time without purposefully targeting the strength deficits in the former.

Henriksson and colleagues (76) shed needed light on the role of youth muscular strength and fitness on later disability in males and opened wide an opportunity for additional longitudinal research. Future prospective trials are needed to examine the link between muscular weakness and disability in females and to determine if youth resistance training interventions could attenuate the risk of disability later in life and reduce health care costs. Such insights will help to inform the development of physical activity guidelines that are consistent with the needs of modern-day youth and advance our understanding of the economic consequences of pediatric dynapenia. Notably, a modest increase in the proportion of youth who participate daily in MVPA could avert billions of dollars in direct medical costs and lost productivity over the course of their lifetimes (92). 


\title{
Physical Activity and Bone Strength During Youth
}

\author{
Experts: Kathleen F. Janz, University of Iowa, and Fátima Baptista, Universidade de Lisboa
}

\begin{abstract}
A behavior's impact on public health is often conceptualized using a population-attributable risk fraction. This concept considers the prevalence of the behavior and the strength of its association (relative risk) to a health outcome. Together, prevalence and relative risk define impact.

Whether you are making the count by participation or by spectatorship, soccer is the most popular sport in the world. It is played in nearly all countries and dominates in Africa, Europe, and South America $(59,79)$. As solid as its ranking is as our number 1 world sport, any count of actual participation is likely low due to the soccer played informally on urban streets, rural fields, and other open spaces that do not lend themselves to a tally. Given soccer's high prevalence, it is time to better understand its
\end{abstract}

association to, and ultimately its impact on, health. In the year following the men's World Cup, this commentary focuses on 2 papers that advance our understanding of the impact of soccer, aka world football, to bone strength in youth. In this commentary, we highlight Lozano-Berges et al (97), which we believe is the first systematic review and meta-analysis to exclusively examine the association of soccer to bone strength indicators. Our second selected paper, Larsen et al (91), is a well-designed, fullyimplemented, and carefully-analyzed randomized school intervention that tests the effect of 2 high-intensity exercise groups ([1] small-sided ball games and [2] circuit weight training) to bone strength indicators. The small-sided ball game arm was primarily soccer.

Expert Choice 1: Lozano-Berges G, Matute-Llorente A, González-Agüero A, Gómez-Bruton A, Gómez-Cabello A, Vicente-Rodríguez G, Casajús JA. Soccer helps build strong bones during growth: a systematic review and meta-analysis. Eur J Pediatr. 2018;177(3):295310. doi: 10.1007/s00431-017-3060-3

\begin{abstract}
The aim of this study was to analyze the effects of soccer practice on bone in male and female children and adolescents. MEDLINE, PubMed, SPORTDiscus and Web of Science databases were searched for scientific articles published up to and including October 2016. Twenty-seven studies were included in this systematic review (13 in the meta-analysis). The metaanalysis was performed by using OpenMeta[Analyst] software. It is well documented that soccer practice during childhood provides positive effects on bone mineral content (BMC) and density (BMD) compared to sedentary behaviors and other sports, such as tennis, weightlifting, or swimming. Furthermore, soccer players present higher BMC and BMD in most weight-bearing sites such as the whole body, lumbar spine, hip, and legs. Moreover, bone differences were minimized between groups during prepuberty. Therefore, the maturity status should be considered when evaluating bone. According to meta-analysis results, soccer practice was positively associated with whole-body BMD either in males (mean difference $0.061 ; 95 \%$ CI, 0.042-0.079) or in females (mean difference 0.063; 95\%CI, 0.026-0.099). Conclusion: Soccer may be considered a sport that positively affects bone mass during growth. Pubertal soccer players presented increased bone mass compared to controls or other athletes; however, these bone differences are minimized during the prepubertal stage.
\end{abstract}

\section{Commentary}

Soccer has much to recommend it as a bone-strengthening activity, given bone adapts to dynamic activities that are moderate to high in load, short in duration, nonrepetitive in direction, and applied quickly (167). During soccer, these movement characteristics occur in an ongoing fashion and at multiple player positions. The ability of soccer, and other physical activities, to promote bone health derives from the mechanical and biochemical relationship between muscle contraction and bone loading during movement. Communication within the musculoskeletal system is established through a unidirectional mechanical relationship in which the muscle acts as a pulley and the bone as a lever to move the body, and by a bidirectional biochemical relationship in which the muscle and bone act as secretory organs helping to regulate metabolism and consequently promoting health (20).

In their systematic review, Lozano-Berges et al (97) examine the singular exposure of soccer using a quantified rate of participation (training hours per week) to DXA-measured whole body, lumbar spine, leg, and hip bone mass. The limited number of bone outcomes among studies proved advantageous since, unlike previous bone outcome reviews $(86,153,167)$, limiting the exposure and outcome measures allowed the study authors to do a statistical pooling of the studies (ie, meta-analysis). The Lozano-Berges et al (97) meta-analysis included over 500 participants and indicated a positive effect of soccer to whole-body bone mass. Across adolescence, results also indicated positive and similar effects of soccer to bone mass in both males and females. The bone mass differences in several weight-bearing bone sites between soccer players and nonathletes were, however, larger after puberty. This is somewhat a novel finding likely due to the cumulative effect of the years of practice and the synergistic effect of velocity of muscle growth and bone-mineral acquisition. The latter makes sense since, in the musculoskeletal system, peak muscle accretion is followed by peak bone accretion, and both occur after puberty (81). A subtle take-home message of this finding is the need for youth to find osteogenic activities that they are willing to do throughout adolescence. This may be where soccer is uniquely positioned as a public health strategy.

Expert Choice 2: Larsen MN, Nielsen CM, Wulff Helge E, Madsen M, Manniche V, Hansen L, Riis Hansen P, Bangsbo J, Krustup P. Positive effects on bone mineralisation and muscular fitness after 10 months of intense school-based physical training for children aged 8-10 years: the FIT FIRST randomised controlled trial. Br J Sports Med. 2018;52(4):254-260. doi: 10.1136/bjsports-2016-096219

Objectives We investigated whether musculoskeletal fitness of school children aged 8-10 years was affected by frequent intense PE sessions. Design and participants 295 Danish school children aged 8-10 years were cluster randomised to a small-sided ball 
game group (SSG) ( $\mathrm{n}=96$, four schools, five classes), a circuit strength training group (CST) ( $\mathrm{n}=83$, four schools, four classes) or a control group (CON, $\mathrm{n}=116$, two schools, five classes). Intervention SSG or CST was performed 3×40 min/week over 10 months. Whole-body dual-energy X-ray absorptiometry (DXA) scans were used to determine areal bone mineral density (aBMD), bone mineral content (BMC) and lean body mass (LBM). Flamingo balance, standing long jump and 20-m sprint tests were used to determine muscular fitness. Results Analysis of baseline-to-10 months change scores showed between-group differences in favour of the interventions in whole-body aBMD (SSG vs CON: $8 \mathrm{mg} / \mathrm{cm}^{2}, 95 \%$ CI 3 to 13; CST vs CON: $7 \mathrm{mg}$ / $\mathrm{cm}^{2}, 95 \%$ CI 2 to 13, p<0.05) and leg BMC (SSG vs CON: $11 \mathrm{~g}$, 95\% CI 4 to 18; CST vs CON: $11 \mathrm{~g}$, 95\% CI 3 to 18 , p<0.05). SSG had higher change scores in leg aBMD compared with CON and CST (SSG vs CON: $19 \mathrm{mg} / \mathrm{cm}^{2}, 95 \%$ CI 11 to 39, p<0.05; SSG vs CST: $12 \mathrm{mg} / \mathrm{cm}^{2}, 95 \%$ CI 3 to $21, \mathrm{p}<0.05$ ), and CST had higher change scores in whole-body BMC compared with CON (CST vs CON: $25 \mathrm{~g}, 95 \%$ CI 10 to 39, p<0.05). Both training types resulted in higher change scores in postural balance (SSG vs CON: 2.4 fewer falls/min, 95\% CI 0.3 to 4.5, CST vs CON: 3.6 fewer falls/ min, 95\% CI 1.3 to 5.9, $\mathrm{p}<0.05$ ) and jump length (SSG vs CON: $10 \%, 95 \%$ CI 5 to $16 \%$; CST vs CON: $9 \%, 95 \%$ CI 3 to $15 \%$, p<0.05). No between-group differences were observed for sprint performance or LBM ( $>00.05$ ). Conclusions In conclusion, $3 \times 40 \mathrm{~min} /$ week with SSG or CST over a full school year improves bone mineralisation and several aspects of muscular fitness of children aged 8-10 years, suggesting that well-organised intense physical education classes can contribute positively to develop musculoskeletal health in young children.

\section{Commentary}

The second edition of the US Physical Activity Guidelines for Americans (161) was released in late autumn 2018. The guidelines confirm a bone-strengthening physical activity dose for children and adolescents of at least 3 days of the week of playing games, running, turning, or jumping (161). In their randomized school intervention, Larsen and colleagues (91) provide solid evidence of what 3 days a week of bone strengthening could look like and, importantly, what to expect from this dose. When compared to controls in a traditional Danish physical education class, the study authors found improved bone mass and musculoskeletal fitness in their two intervention arms: (1) small-side ball games, in which $75 \%$ of the time the children played soccer, and (2) circuit-strength training. Impressive features of this study which bolster its internal validity included a cluster-randomized controlled design, enrollment of nearly 300 prepubertal children, a 10-month training intervention, investigatorled pre- and postmeasures, individual-level measures of training load via accelerometer, and pre- and poststudy measures of sexual maturity and growth. A result we found particularly interesting was the homogeneity of the favorable bone effects across schools in the small-side game intervention, whereas the circuit weight training intervention effects were more heterogenic among schools. This finding indicated greater generalizability in the small-side game arm and suggested that it is easier to deliver and comply with gamebased interventions when compared to circuit training. In fact, the study authors noted that circuit training (30-s all-out exercise interspersed by 45 -s rest) was delivered only half of the time within each intervention period, whereas the other half of the period was spent playing games with similar intensities as circuit training. We suspect this had to do with keeping the children faithful to the intervention for the entire 10-month study period. Larsen et al (91) provide "gold-standard" support that sports, such as soccer, can be osteogenic in normal, healthy children at a dose already recommended in many federal physical activity guidelines $(39,159$, 161,174). In the end, these papers provide new and solid evidence of the value of soccer as an uncomplicated, low-cost, and wellreceived intervention that impacts bone health in youth.

\title{
Growth, Maturation, and Exercise During Youth
}

\author{
Expert: Jaak Jürimäe, University of Tartu
}

Expert Choice 1: Cumming SP, Brown DJ, Mitchell S, Bunce J, Hunt D, Hedges C, Crane G, Gross A, Scott S, Franklin E, Breakspear D, Dennison L, White P, Cain A, Eisenmann JC, Malina RM. Premier League academy soccer players' experiences of competing in a tournament bio-banded for biological maturation. J Sports Sci. 2018;36(7):757-765. doi: 10.1080/02640414.2017.1340656

Individual differences in the growth and maturation have been shown to impact player performance and development in youth soccer. This study investigated Premier League academy players' experiences of participating in a tournament bio-banded for biological maturation. Players $(\mathrm{N}=66)$ from four professional soccer clubs aged 11 and 14 years and between 85-90\% of adult stature participated in a tournament. Players competed in three 11 vs 11 games on a full size pitch with 25 -min halves. Sixteen players participated in four 15-min focus groups and were asked to describe their experiences of participating in the bio-banded tournament in comparison to age group competition. All players described their experience as positive and recommended the Premier League integrate bio-banding into the existing games programme. In comparison to age-group competitions, early maturing players described the bio-banded games more physically challenging, and found that they had to adapt their style of play placing a greater emphasis on technique and tactics. Late maturing players considered the games to be less physically challenging, yet appreciated the having more opportunity to use, develop and demonstrate their technical, physical, and psychological competencies. Bio-banding strategies appear to contribute positively towards the holistic development of young soccer players.

\section{Commentary}

The selection, development, and progression of youth elite athletes is influenced by growth and maturation (84). Biological maturation is a well-documented predictor of performance and also selection in youth soccer (84). Therefore, a selection bias toward early- maturing boys is evident from 12 to 13 years and increases with age and competitive level in youth soccer (60). This means that more mature youth soccer players with greater body size are more likely to be selected into elite youth soccer systems, and receive more advanced coaching expertise and more match-play time (120). A recent effort to address individual differences in biological 
maturation among youth sport and training is labeled biobanding (134). Biobanding is a process whereby youth soccer players are grouped on the basis of maturational rather than age-based criteria (37). This biobanding appears to be most relevant during puberty (age 10-16 years in boys), when maturity-associated differences in body size, function, and physiological adaptations are greatest (134).

The current investigation by Cumming et al (37) examined the experiences and perceptions of youth soccer players participating in a competitive tournament in which players were grouped by maturational rather than chronological age. Participants from Premier League professional soccer clubs, aged 11 and 14 years and between $85 \%$ and $90 \%$ of adult stature, took part in this biobanding study and competed in three 11 vs 11 games on a fullsize pitch (37). This concept of biobanding is simple and easy to apply by using the percentage of predicted adult stature from current age, stature, and weight of participant and mid-parent stature (134). A select group of 16 soccer players participating in focus groups were asked to describe their experiences of participating in the biobanding tournament in comparison to age-group competition. The groups included 8 players identified as advanced in maturation to their age (maturity $\mathrm{Z}$ score $>0.2$ ) and 8 identified as being delayed in maturity to their age (maturity $\mathrm{Z}$ score $<-0.2)(37)$. The maturity $Z$ scores were calculated on the basis of the participant's percentage of predicted adult stature and age- and sex-specific reference values (37).

Early- and late-maturing players agreed that the process of biobanding helped attenuate maturity-associated differences in body size and function. Furthermore, they recommended the Premier League to integrate biobanding into existing games programs (37). Four topics emerged as central to player experiences and perceptions of the biobanded tournament (37): (1) physical development, (2) technical and tactical development, (3) psychosocial development, and (4) overall experience. According to the player experiences, physical development reflected greater competitive equality, while technical and tactical development considered player experiences in relation to adapting their technical and tactical aspects of play and opportunities to demonstrate technical competence. Psychosocial development reflected player perceptions regarding psychological aspects of learning experience and the opportunity to feel leadership and mentoring. The overall experience of all 16 focus group participants described the biobanding soccer tournament as a positive and welcome addition to their game program (37).

It appeared that when playing with physically-matched, yet older and more experienced players, early-maturing boys are exposed to a greater level of challenge and can no longer rely on their physical dominance. Specifically, early-maturing boys had to adapt their game, emphasizing more on technique, tactics, and teamwork over physicality (37). In contrast, late-maturing players described the biobanded games as less physically demanding and technically challenging than age-group competitions. However, late-maturing boys benefited from having more opportunities to utilize and develop certain technical, physical, and psychological attributes and adopt positions of leadership (37). These results clearly demonstrate that there is a need for an additional concept of matching youth athletes on other bases in addition to age-based matching in youth soccer development programs. The biobanding concept of soccer tournament as used in the Cumming et al (37) study may be another opportunity to address individual differences in biological maturation and could serve as an additional developmental strategy in youth soccer.

It has to be considered that although young soccer players appreciated the benefits of biobanding soccer games, it is evident that they still valued competing within their respective age groups (37). Age-group competitions still have many benefits, including the matching of players according to their basis of experience and training, as well as cognitive social and motor development, which are more closely related to chronological age rather than biological maturation (134). Accordingly, more effective and efficient player development in youth soccer should include the provision of both biobanded and age-group competitions. Cumming et al (37) suggested that biobanded competitions should serve as an adjunct to age-group competition and not as a replacement. Such a "hybrid approach" would retain the benefits of age-group competitions together with simultaneously addressing its limitations (134).

In conclusion, biobanding using a noninvasive indicator of biological maturity status in youth soccer players as currently applied by Cumming et al (37) contributes positively to the multilateral development of youth soccer players. However, an efficient soccer development program should include both biobanded and age-group competitions in order to gain maximal benefits in youth soccer.

Expert Choice 2: Farooq MA, Parkinson KN, Adamson AJ, Pearce MS, Reilly JK, Hughes AR, Janssen X, Basterfield L, Reilly JJ. Timing and decline in physical activity in childhood and adolescence: Gateshead Millennium Cohort Study. Br J Sports Med. 2018;52 (15):1002-1006. doi: 10.1136/bjsports-2016-096933

Background and aim There is a widely held and influential view that physical activity begins to decline at adolescence. This study aimed to identify the timing of changes in physical activity during childhood and adolescence. Methods Longitudinal cohort study (Gateshead Millennium Study) with 8 years of follow-up, from North-East England. Cohort members comprise a socioeconomically representative sample studied at ages 7, 9, 12 and 15 years; 545 individuals provided physical activity data at two or more time points. Habitual total volume of physical activity and moderate-to-vigorous intensity physical activity (MVPA) were quantified objectively using the Actigraph accelerometer over 5-7 days at the four time points. Linear mixed models identified the timing of changes in physical activity across the 8-year period, and trajectory analysis was used to identify subgroups with distinct patterns of age-related changes. Results Four trajectories of change in total volume of physical activity were identified representing $100 \%$ of all participants: all trajectories declined from age 7 years. There was no evidence that physical activity decline began at adolescence, or that adolescent declines in physical activity were substantially greater than the declines during childhood, or greater in girls than boys. One group (19\% of boys) had relatively high MVPA which remained stable between ages 7 and 15 years. Conclusions Future policy and research efforts to promote physical activity should begin well before adolescence, and should include both boys and girls. 


\section{Commentary}

It has been established that regular participation in physical activity (PA), particularly in moderate-to-vigorous physical activity (MVPA), is a prerequisite for the current and future health and development in children and adolescents (84). Usually, the research done in the area of PA during childhood and adolescence has suggested that the levels of PA are rather adequate during childhood and the dramatic decline in PA occurs during puberty, being more marked in girls than boys $(34,58)$. This decline in PA during puberty has been thought to reflect adolescent children as a high-risk group for low PA and its consequences for health in these children (58). In contrast, there are studies that have not found dramatic decline in total PA during pubertal maturation (162). Furthermore, total PA may already be in decline in both sexes around the age of school entry (33). Accordingly, it appears that despite the widely-held concept that PA begins to decline during puberty, there is some evidence to suggest that decline in PA may occur earlier (58). However, there is currently very little longitudinal evidence on the timing of changes in objectively-measured PA across childhood and adolescence (58).

The novelty of the Farooq et al (58) study was that PA of boys and girls was measured longitudinally to describe the timing of changes in PA across childhood and adolescence, and to identify when changes in PA really take place. The level of PA was studied from the age of 7 until 15 years, using accelerometry. Group-based modeling of longitudinal data was performed to identify distinct trajectories of age-related changes in total PA and MVPA (58).
Four trajectories of change in total PA were identified and all trajectories declined from 7 years of age in boys and girls. Furthermore, none of the trajectories of total PA demonstrated initial high levels followed by sharp adolescent declines (58). Trajectories of change in MVPA were more complex and it is interesting to note that the MVPA level in $\sim 19 \%$ of boys was relatively high and stable across the 8 -year study period, while other trajectories for boys and girls demonstrated decline in MVPA during the study period. Again, none of the trajectories of MVPA in either boys or girls were characterized by high and stable childhood data followed by a sharp decrease during adolescence (58). Accordingly, the main implication of the Farooq et al (58) longitudinal study for future research and public health policy in the area of PA is the need for a much greater emphasis on childhood rather than adolescence, in both boys and girls. The current study described when changes in PA took place, while the question of why any changes in PA occurred is also important and needs further research (58). Therefore, the novel approach of trajectory analysis used in the Faroog et al (58) study is recommended for use in further longitudinal studies to investigate changes in PA during growth and maturation.

In conclusion, the Farooq et al (58) study clearly demonstrates that PA is already in decline in most individuals of both sexes during childhood and the decline in PA does not begin at adolescence. Furthermore, it has to be taken into account that the decline in PA may not be uniform, as a small but significant minority of boys maintained relatively high and stable MVPA levels across childhood and adolescence in the Farooq et al (58) investigation.

\title{
Physical Activity and Cognition
}

\author{
Experts: Amanda L. McGowan and Matthew B. Pontifex, Michigan State University
}

This section of Pediatric Exercise Science highlights a subset of papers, published in 2018, providing new perspectives regarding the association between chronic physical activity engagement and cognitive function. While it was difficult to select only a few papers to highlight, given the abundance of high-quality work published in 2018, we narrowed our choices down based on the extent to which we viewed these papers as "must-read" publications for discussion among those interested in research in this area. The critical threshold being, would we present these publications for discussion at a lab meeting or journal club? Together, we feel that these papers provide an opportunity to advance our understanding of the association between physical activity and cognition.

Expert Choice 1: Hillman CH, McAuley E, Erickson KI, Liu-Ambrose T, Kramer AF. On mindful and mindless physical activity and executive function: A response to Diamond and Ling (2016). Dev Cogn Neurosci. 2018; Epub ahead of print. doi: 10.1016/j.dcn.2018. 01.006

Expert Choice 2: Diamond A, Ling DS. Aerobic-exercise and resistance-training interventions have been among the least effective ways to improve executive functions of any method tried thus far. Dev Cogn Neurosci. 2018; Epub ahead of print. doi: 10.1016/j.dcn.2018. 05.001

\section{Commentary}

Although we acknowledge the oddity of highlighting two debating commentaries given that both American (1) and Canadian (118) physical activity directives discuss cognitive health and were released in 2018 along with a host of other high-quality studies published this last year, in our opinion, these commentaries should be required reading for anyone interested in the association between chronic physical activity engagement and cognitive control (also known as executive function). Despite the-at times-irreverent tone adopted in their discourse, these commentaries provide compelling insights into how leading scholars in the field of health neuroscience and cognitive development differently view the same body of literature. In response to a summary of a systematic review of the literature examining the effectiveness of interventions for improving cognitive control in youth (41) (but, see the actual systematic review published as a book chapter [42]), Hillman and other notable colleagues (78) published a commentary challenging a number of claims, to which Diamond and Ling (40) published a commentary in rebuttal. Rather than rehash each of the commentaries' various claims and counterclaims, we highlight a few broader points of contention that we feel are of particular interest.

The key point in dispute is that although chronic physical activity interventions may positively impact cognitive control, Diamond and Ling (40) suggest that improvements in cognitive control induced by physical activity are no better or even less than those improvements observed by other interventions. Indeed, despite a robust body of literature demonstrating small to moderate 
effect sizes for cross-sectional differences in cognition associated with chronic physical activity behaviors and resultant differences in the attribute of aerobic fitness, a number of narrative reviews and meta-analyses published over the last several years and presently in press have observed underwhelming effects of chronic physical activity interventions on cognitive function $(7,43,73,101,146,149)$. A key limitation, however, is the paucity of currently published clinical trials conducting well-designed physical activity interventions with these narrative reviews and meta-analyses also often utilizing strict inclusionary criteria that further reduce the available evidence from which to draw. Perhaps one point that does not come through as saliently in these commentaries is that assuming chronic physical activity interventions are indeed no better than other interventions for enhancing cognitive control, the absence of negative effects for cognition suggest that it is still worth intervening to enhance physical activity behaviors. That is, as physical activity is also associated with a host of other cardiovascular and metabolic benefits, an intervention to enhance physical activity might not only induce the same benefits for cognitive control as other interventions but might also incur these physiological benefits as well.

Relatedly, given the small body of extant literature reporting the effects of these chronic physical activity interventions for enhancing aspects of cognition, we still have a relatively immature understanding of what it is about chronic physical activity engagement that might induce these changes in cognition and, in particular, cognitive control. A supposition brought forward by Diamond and Ling (40) suggests that a number of factors related to physical activity engagement could conceivably relate to changes in cognitive control, independent of changes in neurotrophic factors and brain structure/function-which is itself a point of contention discussed by Hillman and colleagues (78) and Diamond and Ling (40) as to whether neuroimaging and nonhuman animal findings should be discussed alongside studies investigating behavioral outcomes. Furthermore, Diamond and Ling (40) suggest that physical activity interventions might be more effective for enhancing cognitive control operations if they were to specifically incorporate cognitively-engaging activities that required aspects of cognitive control (similar to the idea proposed by Best [19]). That is, the context of the physical activity engagement may provide additional opportunities to develop cognitive control as individuals are provided opportunities to challenge themselves and to develop efficacy in a mentored, supportive environment, such as in sporting-related contexts. Thus, in response to Hillman and colleagues' (78) commentary, Diamond and Ling (40) call for physically-active interventions designed to improve cognitive control, with the focus on promoting psychosocial growth and development instead of focusing upon physical activity with the goal of improving cardiorespiratory fitness.

Interestingly, despite the scholarly debate between these 2 groups concerning how they view the same body of literature, the conclusions of both papers ultimately emphasize that, as a field, we need to return to running more empirical investigations. Collectively, this area of research would be better served by finding a balance between reductionist-focused study designs aimed at identifying mechanisms underlying the physical activity-cognition relationship and translational study designs aimed at real-world applications, such as workplace interventions aimed at employee mental health or school-based interventions aimed at integrating physical activity across academic learning contexts. The inherent contrast between basic science and translational work has drastic implications for the design of interventions and how they are approached, but, ultimately, in combination would better advance the field toward theoretical development and evidence-based interventions.

Expert Choice 3: Walsh JJ, Barnes JD, Cameron JD, Goldfield GS, Chaput J-P, Gunnell KE, Ledouz A-A, Zemek RL, Tremblay MS. Associations between 24 hour movement behaviours and global cognition in US children: a cross-sectional observational study. Lancet Child Adolesc Health. 2018;2(11):783-791. doi: 10.1016/S2352-4642(18)30278-5

Background Childhood and adolescence are crucial periods for brain development, and the behaviours during a typical $24 \mathrm{~h}$ period contribute to cognitive performance. The Canadian 24-Hour Movement Guidelines for Children and Youth recommend at least 60 min physical activity per day, 2 h or less recreational screen time per day, and 9-11 h sleep per night in children aged 811 years. We investigated the relationship between adherence to these recommendations and global cognition. Methods In this cross-sectional observational study, we obtained data from the first annual curated release of the Adolescent Brain Cognitive Development study, a 10-year longitudinal, observational study. Data were collected from 21 study sites across the USA between Sept 1, 2016, and Sept 15, 2017. The participants were 4524 US children aged 8-11 years from 20 study sites. Exposures of interest were adherence to the physical activity, recreational screen time, and sleep duration guideline recommendations. The primary outcome was global cognition, assessed with the NIH Toolbox (National Institutes of Health, Bethesda, MD, USA), which we analysed with multivariable linear mixed effects models to examine the relations with movement behaviour variables. Findings Complete movement behaviour data were available for 4520 participants. The mean number of guideline recommendations met was 1.1 (SD 0.9). Overall, 2303 (51\%) participants met the sleep recommendation, 1655 (37\%) met screen time, and $793(18 \%)$ met the physical activity recommendation. $3190(71 \%)$ participants met at least one recommendation, whereas 216 $(5 \%)$ of participants met all three recommendations. Global cognition was positively associated with each additional recommendation met $(\beta=1.44,95 \%$ CI $0.82-2.07, \mathrm{p}<0.0001)$. Compared with meeting none of the recommendations, associations with superior global cognition were found in participants who met all three recommendations $(\beta=3.89,95 \%$ CI 1.43 to $6.34, \mathrm{p}=0.0019)$, the screen time recommendation only $(\beta=4.25,2.50-6.01, \mathrm{p}<0.0001)$, and both the screen time and the sleep recommendations $(\beta=5.15,3.56-6.74, \mathrm{p}<0.0001)$. Interpretation Meeting the $24 \mathrm{~h}$ movement recommendations was associated with superior global cognition. These findings highlight the importance of limiting recreational screen time and encouraging healthy sleep to improve cognition in children.

\section{Commentary}

Given the recent Canadian 24-hour movement guidelines for children, which now not only provide recommendations for physical activity behaviors but also integrate recommendations for sleep and limiting sedentary behavior and screen time (158), we elected to highlight this publication by Walsh and colleagues (166) as it 
provides insights into the extent to which meeting these recommendations relates to differences in cognition. Capitalizing on the initial release of data from the National Institutes of Health (NIH)funded Adolescent Brain Cognitive Development study, a 10-year longitudinal trial collecting data on children's cognitive function and brain structure/function - as well as a number of behavioral patterns - across 21 sites within the United States, this investigation utilized initial cross-sectional data to examine the potential additive effects meeting these 24-hour recommendations had on cognitive function in children. Some caution is warranted in interpreting these findings given that the nature of the data collection limited the assessment of these health-related behaviors to questionnaires: the Youth Risk Behaviour Survey (23) to assess physical activity behaviors (number of days active for at least $60 \mathrm{~min}$ per day in the last week), the Youth Screen Time Survey (143) to assess screen time (number of hours spent on a typical weekday and weekend on screen-based activities), and the Parent Sleep Disturbance Scale for Children (24) to assess sleep (the number of hours of sleep obtained on most nights). However, the sample size of over 4,500 children enabled parsing out the individual components of the 24-hour movement guidelines as well as combinations of those guidelines as to how meeting those guidelines related to performance on the global cognition construct from the NIH toolbox (Bethesda, MD, USA), which is a composite score of performance across tests of attention, cognitive control, language abilities, memory (episodic and working), and processing speed. Accordingly, while lacking nuance in measurement and construct precision, the overall power of the sample size and the diverse and geographically-stratified sample provide unique opportunities for insight.
Consonant with the growing concerns regarding physical inactivity, only $18 \%$ of the sample reported being physically active each day, whereas $51 \%$ of the sample reported sleeping 9 to 11 hours each night, and $37 \%$ reported accumulating 2 hours or less of daily screen time. The strongest associations with performance on the global cognition measure were observed for meeting either the screen time recommendation alone or both the screen time and sleep recommendations. Importantly, however, performance on the global cognition measure was observed to positively relate to meeting these movement guidelines, with meeting more of the guidelines being associated with superior cognition. Given the advantages presented by the dataset, a natural follow-up question to the analysis presented therein would have been to examine the extent to which these relationships exist utilizing different threshold criteria for achieving/not achieving the guidelines-engaging in physical activity 5 days a week instead of 7 , for instance, to determine if there is some threshold of activity above which there are minimal additional benefits for cognitive function-or examining the extent to which time spent in these various activities related to cognition free of the binary confines of achieving/not achieving the guidelines. Nevertheless, this publication contributes to our understanding of the independent and combined effects of these recommended behaviors on a broad construct of cognition. Given the increasingly sedentary behaviors of children and youth, increasing our understanding of the relationship between health behaviors and related physical health attributes is necessary to understand those factors that are important to optimize cognitive health and effective functioning during development.

\title{
Childhood Obesity, Physical Activity, and Exercise: Childhood Obesity and Asthma-Two Distinct Childhood Diseases With a Common Solution?
}

\author{
Expert: Dan Nemet, Meir Medical Center, Tel Aviv University
}

Expert Choice: Lucas JA, Moonie S, Hogan MB, Evans WN. Efficacy of an exercise intervention among children with comorbid asthma and obesity. Public Health. 2018;159:123-128. doi: 10.1016/j.puhe.2018.02.014

\begin{abstract}
Objectives: Children with comorbid asthma and obesity present with more severe and harder-to-control disease than asthmatic children at healthy weight. Weight loss has been shown to improve asthma symptoms, yet physical activity may be difficult due to exercise-induced bronchospasm. Children with asthma have lower exercise rates than non-asthmatics. The objective of this study was to retrospectively evaluate attrition rates and program outcome measures (Body Mass Index [BMI] and maximum oxygen consumption $\left[\mathrm{VO}_{2} \mathrm{max}\right]$ ) among asthmatic and non-asthmatic participants. Study design: Clinical data were collected from the Healthy Hearts Program, a 12-week nutrition and activity intervention program for children who are overweight, obese, or at risk for heart disease and other conditions, and used for the study. Methods: Intervention data and demographics were obtained from medical records at the Children's Heart Center Nevada. Descriptive statistics, paired t-tests, Cox regression analysis, and analysis of covariance were conducted. Results: The mean age of this population $(N=232)$ was 11 years; $54 \%$ were male, $64 \%$ were Hispanic, and $37 \%$ had asthma. Median time in the program was 9 weeks, and $58 \%$ of the population completed the program. Unadjusted analyses showed significant BMI decreases in asthmatic $(\mathrm{P}=0.002)$ and non-asthmatic $(\mathrm{P}=0.001)$ participants and increases in cardiorespiratory function for asthmatic males and females $(\mathrm{P}=0.003, \mathrm{P}=0.004)$ and nonasthmatic males and females $(\mathrm{P}<0.001$ for both). Asthmatic and non-asthmatic children both had improved exercise intensity $(\mathrm{P}=0.033, \mathrm{P}<0.001)$. Conclusions: This program is both beneficial and practical for obese children with asthma for losing weight and improving cardiorespiratory function.
\end{abstract}

\section{Commentary}

Childhood obesity and asthma are two of the most common chronic pediatric diseases. In adults, obesity has been shown to be linked to adult onset asthma but the association in children has been unclear until recently. Authors of more than a dozen longitudinal studies reported the risk of obesity and incident asthma, while several studies report that obesity increases incident asthma only in a subset of the patients, whereas other studies revealed no effect (88).

In 2018, Lang et al (88), demonstrated in a large retrospective study including data from 507,496 children aged 2 to 17 years, that obesity in children indeed increases the risk of a new asthma diagnosis. They estimated that obesity increased the rate of new asthma diagnoses by more than 6 cases per 1,000 patient years and 
that, "By avoiding obesity, children would reduce their risk for new asthma by $26 \%$ to $38 \%$ " (88, p. 7 ). Moreover, Karachaliou et al (85), in another recent study in a large cohort of Greek children, demonstrated a strong association between presence of asthma symptoms, obesity, and sedentary activities, irrespective of socioeconomic and regional factors.

Obesity is now recognized as a worldwide epidemic and major efforts are made to prevent and treat it (139). Despite some recent reports of progress in slowing pediatric obesity, the latest evidence suggests an increase in obesity, particularly in younger children. The mechanisms responsible for the continuing increase in childhood obesity are not completely understood. However, weight gain clearly results from an imbalance between energy intake and energy expenditure (18).

While low levels of physical activity are associated with obesity, the relationship between asthma diagnosis and level of physical activity is less clear, and more complex. In the presence of uncontrolled asthma, exercise-induced bronchoconstriction may lead to decreased physical activity which is associated with increase in body weight. On the other hand, increased body weight leads to physical activity restriction in asthmatic children. Researchers, now trying to delineate the underlying mechanisms for pediatric obesity-related asthma, have suggested several possible mechanisms, such as truncal adiposity, metabolic abnormalities including insulin resistance, dyslipidemia, hyperleptinemia and low levels of adiponectin, as well as obesity-mediated systemic inflammation (129).

Interestingly, the above suggested mechanisms that link obesity and asthma are notable for the lack of a role of allergy. Obesityrelated asthma in children is probably nonallergic and therefore poorly responsive to our "regular" asthma medications.

The recently published 2018 Physical Activity Guidelines Advisory Committee Scientific Report (124) recommends that children and adolescents aged 6 to 17 years should perform 60 minutes (1 hour) or more of moderate-to-vigorous physical activity (MVPA) each day; also, for the first time, the report demonstrates that regular physical activity provides health benefits to children as young as ages 3 to 5 . Yet, most children today, and especially children with chronic diseases and disabilities, do not meet the current physical activity guidelines.
Physical activity interventions have been shown to reduce obesity, improve fitness, and reduce obesity-related metabolic complications by affecting adipocytokines, inflammatory and growth mediators (114). With a possible common mechanism, treating obesity in the asthmatic obese child may be the first modifiable risk factor for obesity-related asthma, offering an opportunity for primary prevention of asthma.

In the highlighted study, Lucas et al (98) aimed to retrospectively evaluate the effects of a 12-week nutrition and physical activity intervention program in asthmatic and nonasthmatic children who are overweight or obese. Two hundred and thirty-two children were included in the analysis; 86 had asthma and 146 were nonasthmatics. The median time in this pilot program was 9 weeks (out of 12), with a range of 1 to 12 weeks, and $58.2 \%$ of the population completed the program $(n=135)$.

Children with asthma completed the intervention at the same rate as children without asthma. Furthermore, children experienced improved cardiorespiratory function, weight loss, and BMI reduction that was positively correlated to the time spent in the program. Children who participated in the program increased their intensity of physical activity from below a moderate level to moderate-tovigorous intensity. No statistically significant difference was found between children with and without asthma, indicating that an exercise program is realistic and beneficial for children who might experience respiratory distress while exercising, as well as children who do not.

Clearly, to our awareness of the cardiovascular and endocrine implications of childhood obesity, we can now add and recognize the effects of obesity on the pulmonary system.

The poor response that children with obesity and asthma have to medications, combined with the enormous economic burden on healthcare systems from both childhood obesity and asthma, call for better understanding of the underlying mechanisms and possible intervention for both children with obesity who develop asthma, and for those children who become obese because of asthma.

This highlights another important cause to promote physical activity from an early age in ALL children, and recognize exercise as an important therapeutic tool for the treatment of childhood obesity and obesity-related asthma.

\title{
Pulmonary Physiology or Diseases, Exercise, and Training During Youth
}

\author{
Expert: Paolo T. Pianosi, University of Minnesota
}

Expert Choice 1: Bovard JM, Welch JF, Houghton KM, McKenzie DC, Potts JE, Sheel AW. Does competitive swimming affect lung growth? Physiol Rep. 2018;6(15):e13816. doi: 10.14814/phy2.13816

Whether the large lungs of swimmers result from intensive training or genetic endowment has been widely debated. Given that peak lung growth velocities occur during puberty, this study examined if competitive swimming during puberty affected lung growth. Eleven- to fourteen-year-old healthy female competitive swimmers and controls were assessed before (PRE) and after (POST) one swimming season (7.4 \pm 0.5 months). Pulmonary function testing included lung volumes, spirometry, diffusion capacity $\left(\mathrm{D}_{\mathrm{L}, \mathrm{CO}}\right)$, and maximal inspiratory $\left(\mathrm{PI}_{\mathrm{MAX}}\right)$ and expiratory ( $\left.\mathrm{PE}_{\mathrm{MAX}}\right)$ pressures. Ventilatory constraints, including endexpiratory lung volume, expiratory flow limitation, and utilization of ventilatory capacity, were assessed during an incremental cycling test. Swimmers $(n=11)$ and controls $(n=10)$ were of similar age, size, and sexual maturity $(P>0.05)$. However, swimmers compared to controls had a greater total lung capacity (PRE $4.73 \pm 0.73$ vs. $3.93 \pm 0.46$, POST 5.08 \pm 0.68 vs. $4.19 \pm$ $0.64 \mathrm{~L} ; P<0.01$ ), peak expiratory flow (PRE $6.48 \pm 0.92$ vs. $5.70 \pm 0.86$, POST $6.97 \pm 0.84 \mathrm{vs} .6 .00 \pm 0.77 \mathrm{~L} \cdot \mathrm{s}^{-1} ; P=0.03$ ), and $\mathrm{PE}_{\mathrm{MAX}}(P<0.001)$. Although $\mathrm{D}_{\mathrm{L}, \mathrm{CO}}$ was greater in swimmers $(P=0.01)$, differences were attenuated when expressed relative to alveolar volume (PRE $5.14 \pm 0.60$ vs. $5.44 \pm 0.44$, POST $4.91 \pm 0.56$ vs. $\left.5.16 \pm 0.38 \mathrm{~mL} \mathrm{~min}^{-1} \mathrm{mmHg}^{-1} \mathrm{~L}^{-1} ; P=0.20\right)$. The groups achieved a similar maximal oxygen uptake $(P=0.32)$, and ventilatory constraints experienced were not different $(P>$ $0.05)$. Changes over time were not different between groups $(P>0.05)$. At the initial measurement, pubertal female swimmers had greater lung size, expiratory flows, and indices of respiratory muscle strength, but similar ventilatory constraints while 
cycling. One competitive swimming season did not further accentuate this enhanced lung size and function or alter ventilatory mechanics, suggesting that competitive swimming during puberty did not affect lung growth.

\section{Commentary}

Whether the large lungs of swimmers result from intensive training or genetic endowment has been widely debated. Given that peak lung growth velocities occur during puberty, this study (21) examined if competitive swimming during puberty affected lung growth. Eleven- to 14-year-old healthy female competitive swimmers and controls were assessed before (PRE) and after (POST) one swimming season $(7.4 \pm 0.5$ months). Pulmonary function testing included lung volumes, spirometry, diffusion capacity $\left(\mathrm{D}_{\mathrm{L}, \mathrm{CO}}\right)$, and maximal inspiratory $\left(\mathrm{PI}_{\mathrm{MAX}}\right)$ and expiratory $\left(\mathrm{PE}_{\mathrm{MAX}}\right)$ pressures. Ventilatory constraints, including end-expiratory lung volume, expiratory flow limitation, and utilization of ventilatory capacity, were assessed during an incremental cycling test. Swimmers $(n=11)$ and controls $(n=10)$ were of similar age, size, and sexual maturity $(P>.05)$. However, swimmers compared to controls had a greater total lung capacity (PRE $4.73 \pm 0.73$ vs $3.93 \pm$ 0.46 , POST $5.08 \pm 0.68$ vs $4.19 \pm 0.64 \mathrm{~L} ; P<.01$ ), peak expiratory flow (PRE $6.48 \pm 0.92$ vs $5.70 \pm 0.86$, POST $6.97 \pm 0.84$ vs $6.00 \pm$ $\left.0.77 \mathrm{~L} \cdot \mathrm{s}^{-1} ; P=.03\right)$, and $\mathrm{PE}_{\mathrm{MAX}}(P<.001)$. Although $\mathrm{D}_{\mathrm{L}}$, co was greater in swimmers $(P=.01)$, differences were attenuated when expressed relative to alveolar volume (PRE 5.14 \pm 0.60 vs $5.44 \pm 0.44$, POST $4.91 \pm 0.56$ vs $5.16 \pm 0.38 \mathrm{~mL} \cdot \mathrm{min}^{-1}$. $\mathrm{mmHg}{ }^{-1} \cdot \mathrm{L}^{-1} ; P=.20$ ). The groups achieved a similar maximal oxygen uptake $(P=.32)$, and ventilatory constraints experienced were not different $(P>.05)$. Changes over time were not different between groups $(P>.05)$. At the initial measurement, pubertal female swimmers had greater lung size, expiratory flows, and indices of respiratory muscle strength, but similar ventilatory constraints while cycling. One competitive swimming season did not further accentuate this enhanced lung size and function or alter ventilatory mechanics, suggesting that competitive swimming during puberty did not affect lung growth.

It has been appreciated for decades that swimmers as a group have above average lung volumes (forced vital capacity [FVC], total lung capacity [TLC]) (15). This observation begs the hallowed question in the title of this chosen manuscript (21), which has been addressed repeatedly over the years in the respiratory physiology literature. The fact that debate continues implies there are studies supporting both positions. A corollary question is often asked in clinical encounters with pulmonary patients. Parents are understandably interested in any treatment that augments lung growth, particularly preterm infants whose lungs are typically underdeveloped.

In order to address this, one must first understand a modicum of pulmonary function testing. Tests measuring static and dynamic lung volumes require that subjects understand instructions, follow directions, and be able to sustain exhalation for several seconds. This ability is generally attained by age 7 years, but the period of maximal lung growth very likely occurs before this age, which poses a challenge to address the titular question. Chest wall dimensions increase at a more or less constant rate from age 6 until age 14 to 15 in girls (145). The timing of peak somatic growth velocity precedes that of maximal lung functional growth and the early growth spurt in females includes the lungs (144). However, lung function continues to grow after attainment of final adult height, at least in boys (77).

These observations were used to justify the approach of investigators who reasoned that 11- to 14-year-old female competitive swimmers and healthy female controls of similar age, size, and maturation should undergo detectable growth in static and dynamic lung volumes over a finite period. They compared initial differences in lung size and function and monitored these variables to determine if one season of competitive swimming affected the development of lung size and function. Controls participated primarily in gymnastics, dance, and team sports, but did not perform any sport-specific endurance training. Physical activity levels were self-assessed with a modified version of a validated physical activity questionnaire. Self-reported daily moderate-vigorous physical activity $(121 \pm 25$ vs $110 \pm 55 \mathrm{~min})$ and physical activity levels based on this score were not different between groups. Subjects self-assessed sexual maturity using a validated form corresponding to Tanner stages.

Authors found that prior to a season of training, swimmers already had more robust lung function relative to both predictive values and compared with controls. The greater lung size (ie, TLC) and function (ie, expiratory flow rates) occurred regardless of the starting age of swimming or years of swimming experience. Furthermore, the changes in lung size and function during one competitive swimming season were similar between swimmers and controls. Mean growth was not different between swimmers and controls: TLC $(0.35 \pm 0.13 \mathrm{~L}$ [SWIM] vs $0.26 \pm 0.25 \mathrm{~L}[\mathrm{CON}]$ or $8 \pm$ $4 \%$ [SWIM] vs $6 \pm 6[\mathrm{CON}]$ ); FVC $0.23 \pm 0.15 \mathrm{~L}$ (SWIM) vs $0.15 \pm 0.19 \mathrm{~L}(\mathrm{CON})$. Percent-predicted values did not change from during the course of a single season in swimmers or controls, suggesting that both groups experienced normal lung growth for the age and somatic growth. These changes occurred irrespective of the training volume in swimmers. Pubertal female swimmers already have greater lung size and function compared to controls but these results support the notion that competitive swim training during periods of peak lung growth does not influence lung development.

In essence, swimmers bring it to the sport rather than participation in the sport stimulates lung growth. There are innumerable benefits to participation in vigorous exercise training programs (see next) but, unfortunately, stimulation of lung growth is not one of them. That said, this study was done in healthy controls, and while I venture to say the same would hold true in patients with lung disease, this is only speculation. As far as we know, there are only 2 factors that stimulate lung growth: chronic hypoxia (eg, living at altitude) and lung resection (164).

Expert Choice 2: Radtke T, Hebestreit H, Gallati S, et al. CFTR genotype and maximal exercise capacity in cystic fibrosis. A crosssectional study. Ann Amer Thoracic Soc. 2018;15(2):209-216. doi: 10.1513/AnnalsATS.201707-570OC

Rationale: Cystic fibrosis transmembrane conductance regulator (CFTR) is expressed in human skeletal muscle cells. Variations of CFTR dysfunction among patients with cystic fibrosis may be an important determinant of maximal exercise capacity in cystic fibrosis. Previous studies on the relationship between CFTR genotype and maximal exercise capacity are scarce and contradictory. Objectives: This study was designed to explore factors influencing maximal exercise capacity, expressed as 
peak oxygen uptake $\left(\dot{\mathrm{V}}_{2 \text { peak }}\right)$, with a specific focus on $C F T R$ genotype in children and adults with cystic fibrosis. Methods: In an international, multicenter, cross-sectional study, we collected data on CFTR genotype and cardiopulmonary exercise tests in patients with cystic fibrosis who were ages 8 years and older. CFTR mutations were classified into functional classes I-V. Results: The final analysis included 726 patients (45\% females; age range, 8-61 yr; forced expiratory volume in $1 \mathrm{~s}, 16$ to $123 \%$ predicted) from 17 cystic fibrosis centers in North America, Europe, Australia, and Asia, all of whom had both valid maximal cardiopulmonary exercise tests and complete CFTR genotype data. Overall, patients exhibited exercise intolerance $\left(\mathrm{V}_{2 \text { peak }}\right.$, $77.3 \pm 19.1 \%$ predicted), but values were comparable among different CFTR classes. We did not detect an association between CFTR genotype functional classes I-III and either $\dot{\mathrm{V}}_{2 \text { peak }}$ (percent predicted) (adjusted $\beta=-0.95 ; 95 \% \mathrm{CI},-4.18$ to $2.29 ; P=$ 0.57 ) or maximum work rate (Watt ${ }_{\max }$ ) (adjusted $\beta=-1.38 ; 95 \% \mathrm{CI},-5.04$ to $2.27 ; P=0.46$ ) compared with classes IV-V. Those with at least one copy of a F508del-CFTR mutation and one copy of a class V mutation had a significantly lower $\dot{V}_{2 \text { peak }}(\beta=$

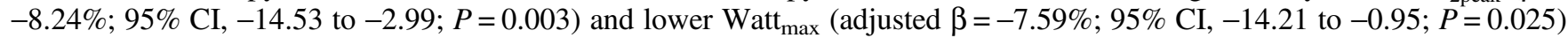
than those with two copies of a class II mutation. On the basis of linear regression analysis adjusted for relevant confounders, lung function and body mass index were associated with $\mathrm{VO}_{2 \text { peak. }}$. Conclusions: $C F T R$ functional genotype class was not associated with maximal exercise capacity in patients with cystic fibrosis overall, but those with at least one copy of a F508del-CFTR mutation and a single class $\mathrm{V}$ mutation had lower maximal exercise capacity.

\section{Commentary}

Cystic fibrosis (CF) is a common inherited multi-system disease in the white population predominantly of Northern European origin. It is caused by a mutation in CF transmembrane regulator (CFTR), an epithelial chloride channel present in many tissues including sarcoplasmic reticulum in skeletal muscle cells and myocardium. There are 5 classes of mutation whose net result ranges from no protein production (class I) to production of a protein with partial function (classes IV or V). Like sickle cell disease, it is inherited as an autosomal recessive disease, but, unlike sickle cell disease, no genetic advantage to heterozygosity (carrying only 1 copy of the gene and therefore free of disease) has been shown. It is generally accepted that disease modifiers, possibly genetic, exist to explain phenotypic variability among patients with identical genotypes (45) but few have been identified (72). It is well known that aerobic fitness, expressed as peak $\dot{V} O_{2}$, is associated with longevity in patients with $\mathrm{CF}(115,123)$, and there is a genetic component to aerobic fitness (140). Such findings may prompt one to speculate whether there is a genetic association between aerobic fitness and CFTR mutation, presumably beneficial. Indeed, 1 study showed CF patients with 1 copy of the most common mutation (F508del, a class II mutation) had significantly better exercise capacity if the second mutation was class IV or $\mathrm{V}$, where a partially functioning protein is made and occupies its niche at the epithelial apical membrane (142).

On this background, Radtke et al (127) collated results of exercise tests from CF clinics in Europe, Asia, North America, and Australia and assembled a cross-sectional snapshot of exercise tolerance and its relationship with CFTR genotype in approximately 800 patients. Now, the devil is in the details (found in their online supplement), but these authors (127) did an admirable job accounting/correcting for potentially confounding variables. For example, infection with Ps. aeruginosa is associated with steeper decline in peak $\dot{V} O_{2}$ over time (163). They analyzed peak $\dot{V} O_{2}$ and peak work capacity (PWC) as percent predicted values (problematic in and of itself), but also performed linear mixed models using a multiplicative, allometric approach to account for potential effects of body size and pulmonary function on the relationship between CFTR genotype and peak $\dot{V} \mathrm{O}_{2}$.

In short, this manuscript confirmed previously observed correlations from various centers $(87,100)$. Peak $\dot{V} O_{2}$ is significantly associated with:

- nutritional status,

- pulmonary function,

- but not CFTR mutation.
Furthermore, in mixed models, the presence of $P S$. aeruginosa infection was associated with lower peak $\dot{V} O_{2}$ and PWC. In a mixed-effects models, they found no association between CFTR genotype functional class and either peak $\dot{V} O_{2}$ or PWC (percent predicted). In these models, both lung function and nutritional status were associated with peak $\dot{V} \mathrm{O}_{2}$ and PWC, independent of CFTR mutation. These results were confirmed when allometric modeling was applied and when exercise capacity was expressed per kilogram of body weight or lean body mass. Results were similar when the 112 patients with nonmaximal CPETs (defined in online supplement) were included in the analysis.

This paper should lay to rest the notion that CFTR genotype affects exercise performance measured as peak $\dot{V} O_{2}$. It is by far the largest series of its kind, and the authors carefully vetted data from many different centers and performed thorough analyses, taking into account principal (but obviously not all) potentially confounding variables. In my humble opinion, this paper should prompt $\mathrm{CF}$ clinics not only to perform annual or biennial peak exercise tests on their patients (74), but also monitor physical activity with validated measures at the same time (75). Information so gained is as important as microbiology and spirometry in appreciating the evolution of functional status, limitation, and disease progression.

\section{Conclusion and Speculation}

These papers selected illustrate that (a) regular and vigorous aerobic exercise do not improve lung capacity and (b) the respiratory system is a likely bottleneck in the pathway for oxygen and (one way or another) limits exercise in patients with cystic fibrosis. These results should really not come as a surprise when one understands the interconnectedness of the different cogs in the wheels depicting the pathway for oxygen. Although one may be able to do more work for the same level of ventilation in the trained versus untrained state, this has nothing to do with lung capacity(ies). Only 2 items thus far have been shown to stimulate lung growth: living at high altitude and lung resection (though beyond what age of life this ceases remains speculative). Indeed, Wagner's (164) persuasive argument behooves us to challenge conventional wisdom based on (flawed) empiricism.

As illustrated by Wagner (164), the pathway for oxygen is a series of conductances (inverse of resistance) in $\mathrm{mL} \cdot \mathrm{s}^{-1} \cdot \mathrm{cmH}_{2} \mathrm{O}^{-1}$, with the size of the conductance proportional to its magnitude. Regular training increases stroke volume such that peak cardiac output is higher, improves mitochondrial density and enzyme activities (83), and increases muscle blood flow regulation and distribution (38). In other words, exercise enhances every component of the $\mathrm{O}_{2}$ transport system except the lungs (164). 


\title{
Immunology and Exercise During Youth
}

\author{
Experts: Shlomit Radom-Aizik and Nitzan Dror, University of California Irvine
}

Expert Choice: Morgado JP, Monteiro JP, Matias CN, Reis JF, Teles J, Laires MJ, Alves F. Long-term swimming training modifies acute immune cell response to a high-intensity session. Eur J Appl Physiol. 2018;118(3):573-583. doi: 10.1007/s00421-017-3777-8

\begin{abstract}
Purpose Long-term training influence on athletes' immune cell response to acute exercise has been poorly studied, despite the complexity of both chronic and acute adaptations induced by training. The purpose of the study is to study the influence of a 4month swimming training cycle on the immune cell response to a high-intensity training session, during $24 \mathrm{~h}$ of recovery, considering sex, maturity, and age group. Methods Forty-three swimmers (16 females, $14.4 \pm 1.1$ years; 27 males, $16.2 \pm 2.0$ ) performed a standardized high-intensity session, after the main competition of the first (M1), and second (M2) macrocycles. Blood samples were collected before (Pre), immediately after (Post), $2 \mathrm{~h}$ after (Post2h) and $24 \mathrm{~h}$ after (Post24h) exercise. Haemogram and lymphocytes subsets were assessed by an automatic cell counter and by flow cytometry, respectively. Subjects were grouped according to sex, competitive age groups, or pubertal Tanner stages. Results express the percentage of relative differences from Pre to Post, Post2h and Post24h. Upper respiratory symptoms (URS) and training load were quantified. Results At M2, we observed smaller increases of leukocytes (M1: 14.0 $\pm 36.3 / \mathrm{M} 2: 2.33 \pm 23.0 \%)$ and neutrophils (M1: 57.1 \pm 71.6/M2: $38.9 \pm 49.9 \%)$ at Post; and less efficient recoveries of total lymphocytes $(\mathrm{M} 1:-22.0 \pm 20.1 / \mathrm{M} 2:-30.0 \pm 18.6 \%)$ and CD19+ $(\mathrm{M} 1: 4.09 \pm 31.1 / \mathrm{M} 2:-19.1 \pm 24.4 \%)$ at Post $2 \mathrm{~h}$. At Post $2 \mathrm{~h}$, the increment of CD4 ${ }^{+} / \mathrm{CD}^{+}$was smaller in youth $(\mathrm{M} 1: 21.5 \pm 16.0 /$ M2: $9.23 \pm 21.4 \%$ ), and bigger in seniors (M1: $3.68 \pm 9.21 / \mathrm{M} 2: 23.2 \pm 15.0 \%$ ); and at Post24h late pubertal swimmers' $\mathrm{CD}_{16}{ }^{+} 56^{+}$recovered less efficiently $(\mathrm{M} 1:-0.66 \pm 34.6 / \mathrm{M} 2:-20.5 \pm 34.2 \%)$. Conclusions The training cycle induced an attenuated immune change immediately after exercise and a less efficient recovery of total lymphocytes, involving an accentuated $\mathrm{CD}_{1} 9^{+}$decrease. The concomitant higher URS frequency suggests a potential immune depression and a longer interval of susceptibility to infection.
\end{abstract}

\section{Commentary}

Research into exercise immunology has advanced over the past 3 decades, with an emphasis on understanding how different modes, volumes, durations, and intensities of exercise affect immune function. While regular moderate exercise results in improved immune function and decreased risks of disease susceptibility (172), extensive overtraining may lead to immune suppression and increased risks of disease susceptibility. Initial interest in exercise immunology was driven primarily by anecdotal evidence from athletes and coaches indicating that periods of intense training were associated with an increased frequency of illness and infection (155). The field grew rapidly with the seminal discoveries that exercise stimulates a wide variety of immune and inflammatory mediators (25). Very little is known regarding the interactions of acute and chronic exercise and the immune system in children and adolescents and their implications for athletic performance. Due to lack of information, advice regarding exercise and infection offered to the young athlete is based on adult data, which may not be accurate or even appropriate for the child (155).

We chose to highlight an important work by Morgado et al (110), who studied the immune system response, using flow cytometry, to an acute bout of exercise during a "real-life" swimming season (total of 30 weeks) in adolescents of different maturity stages. Forty-three swimmers participated in a 4-month training cycle of a swimming season. Training load and mean intensity of all scheduled swimming sessions were quantified, physiological characteristics were assessed, and incidence of upper respiratory symptoms (URS) was monitored throughout the follow-up season. To evaluate the acute effect of exercise on the immune system in 2 different time points during the season (after the main competitions of the first and second macrocycles), blood was drawn before, immediately after, 2 hours, and 24 hours into recovery from a standardized high-intensity swimming session.

Overall, an attenuated acute immune response was observed in the later and more intense period of the training season, with smaller increase in leukocytes and neutrophils following the acute exercise challenge and less efficient recovery of the total lymphocytes and B cell lymphocytes, demonstrated by higher magnitude of the decrease from Pre to Post2h of total lymphocytes and CD19 ${ }^{+}$ subset. Concurrently, there was a higher URS frequency during the later period of the training, which supports the idea of a potential immune depression and a longer interval of immune susceptibility to infection as a result of the cumulative effects of the swim training loads, although a seasonal effect cannot be ruled out.

Age-related changes in the immune system throughout childhood have been studied extensively, with a particular focus on childhood infectious diseases and vaccination science (32). Despite this, very little research has focused on the role of exercise in modulating the immune system in the growing child $(31,156)$. It is likely that exercise effects are tied to the interactive mechanisms of energy balance (ie, diet, physical activity, and energy metabolism) which collectively can modify, and be modified by, immune mediators $(50,65,119)$. Morgado et al (110) showed in the later period of training, age-dependent immune response reflected by lower magnitude of change in the youngest group in the $\mathrm{CD}^{+} / \mathrm{CD}^{+}$ratio (T helper cells to cytotoxic T cells) 2 hours into recovery, and maturity-dependent immune response reflected by less efficient recovery of NK cells in the late-pubertal group compared to the group who had completed puberty. The authors conclude that the younger athletes' more attenuated immune responses can make them more sensitive to infection during a long-term intense training, and highlight the importance of controlling for age-related variables, especially during adolescence.

An improved understanding of normal immunological responses to acute exercise in healthy children and adolescents is critical if we are to design evidence-based exercise prescriptions and programs for both healthy children and those with chronic diseases. The particular constellation of novel technologies (eg, flow cytometry), data analytics (eg, machine learning), and the emergence of systems biology (132) position researchers to make unprecedented advances in this poorly studied field. 


\title{
Cardiovascular Physiology and Disease During Youth
}

\author{
Expert: Thomas Rowland, Skidmore College
}

Expert Choice 1: Wittekind SG, Gerdes Y, Mays W, Chin C, Jefferies JL. Cardiac rehabilitation improves cardiometabolic health in young patients with nonischemic dilated cardiomyopathy. Texas Heart Inst J. 2018;45(1):27-30. doi: 10.14503/THIJ-17-6249

\begin{abstract}
Nonischemic dilated cardiomyopathy is deadly and costly, and treatment options are limited. Cardiac rehabilitation has proved safe and beneficial for adults with various types of heart failure. Therefore, we retrospectively evaluated the hypothesis that rehabilitation is safe and improves cardiometabolic health in young patients with nonischemic dilated cardiomyopathy. From 2011 through 2015, 8 patients (4 males) (mean age, $20.6 \pm 6.6 \mathrm{yr}$; range, 10-31 yr) underwent rehabilitation at our institution. They were in American Heart Association class C or D heart failure and were on maximal medical therapy. Their mean left ventricular ejection fraction at baseline was $0.26 \pm 0.15$. Two patients had a left ventricular assist device, and 2 were inpatients. To evaluate safety, we documented adverse events during rehabilitation sessions. Clinical endpoints were measured at baseline, immediately after completing rehabilitation, and after one year. Patients attended 120 of 141 possible sessions (85\%), with no adverse events. There were no marked changes in mean left ventricular ejection fraction or body mass index. The patients' mean waist circumference decreased by $1.37 \pm 0.6$ in $(n=5 ; 95 \% \mathrm{CI},-2.1$ to -0.63$)$. Their 6 -minute walk distance increased by a mean of $111 \pm 75 \mathrm{~m}(\mathrm{n}=5 ; 95 \% \mathrm{CI}, 18-205)$. In our small sample of young patients with nonischemic dilated cardiomyopathy, cardiac rehabilitation was feasible and was associated with minimal risk. Our findings suggest that prospective studies in this population are warranted.
\end{abstract}

\section{Commentary}

This report (171) details the outcomes of an organized 16-week cardiac rehabilitation program for a small number of young patients with serious myocardial dysfunction and clinical findings of congestive heart failure from dilated cardiomyopathy. While the patients experienced favorable improvements in exercise capacity without adverse effects, this study can be interpreted from a broader perspective as addressing 2 important questions: First, is exercise, or physical activity, beneficial to children with significant heart disease? Second, what are the effects of exercise-positive or negative-on heart muscle function in children with myocardial dysfunction?

The answer to the first question is a resounding "yes." There is no argument that engaging in regular exercise is important for the well-being of the general pediatric population from many standpoints_-physical and mental health, building social relationships, and the development of motor skills-besides the contribution that being physically active makes to the enjoyment of life. Youth with heart disease need to share in these benefits. Unfortunately, because of fear or ignorance or overprotection, many do not (99). We need to find effective means of encouraging children with cardiac defects to participate in regular physical activity, even those whose disease places physical constraints that limits that activity.

The second question, concerning the effect of exercise on a dysfunctional heart muscle, is more complicated and equally important, but, unfortunately, comes with no simple answer. This issue has become more pertinent with the recent development of surgical techniques which have permitted the survival of infants with complex congenital heart disease. These are anomalies which once caused death in the newborn period but, today, as a consequence of surgical advances, allow long-term survival. Most of these involve a heart with only one functioning ventricle, the other being severely underdeveloped (eg, tricuspid atresia, hypoplastic left ventricle). While most of these children are now surviving following surgery, they suffer from a number of ongoing problems, one of which is myocardial dysfunction. The question, then, surrounds the problem confronting the physician in counseling these young patients: Is exercise beneficial or injurious to a poorly functioning heart muscle? Should restrictions be placed on levels of physical activity? Or, to the contrary, should exercise be encouraged? The question is thus a very real-world practical one.
Intuitively, one would think that provoking increased heart work with exercise would be injurious to a myocardium that is already dysfunctional due to excessive work caused by the cardiac anomaly. Conditions besides heart disease can cause myocardial dysfunction and even congestive heart failure in the situation of prolonged work, such as obesity, anemia, arterial-venous malformations, pregnancy, tachycardia, and ultra-endurance athletic events. On the other hand, it might be argued that positive functional adaptations created by appropriate stress is a hallmark of human physiology. Might such adaptive responses to exercise serve to improve heart muscle function in these patients?

Unfortunately, the number of research investigations which have examined the effect of exercise on cardiac function in young patients with myocardial dysfunction - the means of answering this question - are meager (48). That is why this study by Wittekind et al (171) is particularly valuable, even if it involved only 8 patients whose myocardial dysfunction was either idiopathic or related to anthracycline toxicity. Even though certain improvements in quality of life and exercise tolerance were observed, there was no significant change in myocardial contractility, as measured by the left ventricular ejection fraction $(0.26 \pm 0.17$ prior to the exercise program, $0.28 \pm 0.17$ at its conclusion).

The extant research literature in youth is meager. Duppen et al described magnetic resonance imaging (MRI) and tissue Doppler imaging measures of resting left ventricular function in 2 studies of children and young adults with corrected tetralogy of Fallot or Fontan circulation before and after a 12-week endurance training program $(46,47)$. No changes were observed. Somarriba et al found no changes in resting echocardiographic shortening fraction of the left ventricle in 2 young patients with dilated cardiomyopathy after a similar exercise program (150).

There is much more information about the effects of exercise rehabilitation on myocardial function in adults following surgery for coronary artery disease. In general, these have demonstrated findings similar to the scant pediatric literature cited above: improvements in quality of life, better long-term survival, and enhanced exercise tolerance, but minor or no changes in myocardial function. No obvious detrimental effects of exercise were evident in these programs. One must keep in mind that the physiology is different in these adults than that of children with congenital heart disease, reflecting dysfunction from myocardial 
ischemia rather than overwork of congenital heart disease. So whether or not such results in an older population are relevant to children with postoperative heart disease is unclear.

One is left, then, with making judgments regarding the wisdom of exercise in young patients with myocardial dysfunction on a very limited scientific basis. Despite theoretical considerations, however, there appears to be no clear evidence at present that regular endurance exercise, recreational or as part of a rehabilitation program, either improves or depresses contractile capacity in these patients. The corollary, then, would be that given the noncardiac benefits of exercise rehabilitation, regular physical activity - within their individual functional limits-can be promoted for these children. It is important to recognize, though, that other considerations, particularly susceptibility to serious dysrhythmias, need to be included in such decisions.

The more definitive conclusion, though, is that this is an important issue, and we are in need of much more research information. Newer methods of assessing myocardial dysfunction noninvasively during exercise have recently been developed (135). We can expect, then, in the future we should be able to much better address this question of the influence of exercise in patients with myocardial dysfunction.

Expert Choice 2: Murali M, Hofman PL, Derraik JGB, Cutfield WS, Hornung T, Gusso S. Exercise capacity and cardiac function in adolescents born post-term. Sci Rep. 2018;8(1):12963. doi: 10.1038/s41598-018-31343-3

There is some evidence that children born post-term ( $\geq 42$ weeks of gestation) have metabolic abnormalities that may be associated with an increased risk of adverse health outcomes in adulthood. However, there are no data as to whether adolescents born post-term display alterations in aerobic capacity or cardiovascular function. We studied 48 adolescents (56\% males) in Auckland (New Zealand) with a mean age of 14.3 years (SD =1.7): 25 born post-term and 23 born at term (37-41 weeks of gestation). Assessments included metabolic markers in blood, whole body DXA scans, 24-hour ambulatory blood pressure monitoring, maximal exercise capacity, as well as cardiac MRI scan at rest and during submaximal exercise. Exercise capacity was lower in the post-term than in control participants ( $44.5 \mathrm{vs} 47.8 \mathrm{ml} / \mathrm{kgffm} / \mathrm{min} ; \mathrm{p}=0.04)$. There were no differences in left ventricular volumes at rest and during exercise between groups. The 24-hour ambulatory blood pressure monitoring also showed no differences between the two groups. Being born post-term was associated with reduced exercise capacity, but with no observed differences in central cardiac function. We speculate that the reduction in exercise capacity may be due to changes in the peripheral vascular system.

\section{Commentary}

Traditionally, babies who are born postterm ( $\geq 42$ weeks) and large for gestational age (over $8 \mathrm{lb} 13 \mathrm{oz}[\sim 3.7 \mathrm{~kg}]$ ) have been recognized to face certain increased risks in the immediate postpartum period, including hypoglycemia from hyperinsulinemia, meconium aspiration, transit hypertrophic cardiomyopathy, and misadventures of trauma during delivery. Recently, follow-up studies have provided evidence that these babies may also exhibit long-term health issues as well, such as obesity, metabolic syndrome, and reduced insulin sensitivity. This study by Murali et al. (111) is important in being the first to explore the potential impact of prenatal factors resulting in macrosomia and prolonged gestation on cardiovascular fitness.

The central finding here was that the adolescents who had been born postterm demonstrated a lower level of aerobic fitness on a standard progressive cycle test to exhaustion compared to control participants. This study was well-designed, and the lower $\mathrm{VO}_{2} \mathrm{max}$ in the postterm group (by an average of $3.3 \mathrm{ml} / \mathrm{kg}$ fatfree mass) could not be explained by differences in body composition, habitual physical activity, socioeconomic status, or test motivation (identical values of maximal heart rate). Blood pressure and workload levels were similar between the 2 groups. So, what was responsible for the lower aerobic fitness? More particularly, what effects of the longer in-uterine stay (mean 42.1 vs 39.3 weeks) and greater birth weight (mean 3938 vs 3552 grams) in the postterm participants could account for this difference?
The authors performed cardiac anatomic and functional measurements at rest and during mild submaximal supine exercise (to an average heart rate of $115 \mathrm{bpm}$ ) and could find no group differences in left ventricular mass, contractility (ejection fraction), cardiac output, stroke volume, or end-diastolic or end-systolic ventricular dimensions. Based on this, they concluded that the lower adjusted $\mathrm{VO}_{2}$ max in the postterm participants could not be explained by any differences in cardiac functional reserve. Of course, this was not true, as cardiac variables were not measured at maximal exercise. So, it remains possible that there existed some cardiac-related contribution to the depressed maximal aerobic capacity. Such a mechanism is not implausible, given that insulin resistance in adolescents with type 1 diabetes has been shown to negatively affect cardiovascular function (112).

Instead, the authors concluded that the reduction in aerobic fitness "may be due to changes in the peripheral vascular system" (111, p. 1) This, they suggested, might be linked to a decreased peripheral insulin sensitivity, reducing "capillary vasodilation" and thereby altering peripheral oxygen extraction. As both maximal cardiac reserve function and oxygen extraction were not measured in this study, such ideas remain speculative.

The importance of this study is not in revealing (or not) any responsible mechanism for the lower aerobic fitness in postterm adolescents but rather in contributing to the recognition that intrauterine influences may have profound influences on the future health of these babies. Does this include negative influences on oxygen delivery and/or utilization? We await future investigations to verify such an effect, as well as to provide insights into its mechanism.

\title{
Physical Activity, Inactivity, and Health During Youth
}

\author{
Expert: Alex V. Rowlands, University of Leicester
}

Expert Choice: Aadland E, Kvalheim OM, Anderssen SA, Resaland GK, Andersen LB. The multivariate physical activity signature associated with metabolic health in children. Int J Behav Nutr Phys Act. 2018;15:77. doi: 10.1186/s12966-018-0707-z 
Background: Physical activity is a cornerstone for promoting good metabolic health in children, but it is heavily debated which intensities (including sedentary time) are most influential. A fundamental limitation to current evidence for this relationship is the reliance on analytic approaches that cannot handle collinear variables. The aim of the present study was to determine the physical activity signature related to metabolic health in children, by investigating the association pattern for the whole spectrum of physical activity intensities using multivariate pattern analysis. Methods: We used a sample of 841 children (age $10.2 \pm 0.3$ years; BMI 18.0 $\pm 3.0 ; 50 \%$ boys) from the Active Smarter Kids study, who provided valid data on accelerometry (ActiGraph GT3X+) and several indices of metabolic health (aerobic fitness, abdominal fatness, insulin sensitivity, lipid metabolism, blood pressure) that were used to create a composite metabolic health score. We created 16 physical activity variables covering the whole intensity spectrum (from 0-100 to $\geq 8000$ counts per minute) and used multivariate pattern analysis to analyze the data. Results: Physical activity intensities in the vigorous range (5000-7000 counts per minute) were most strongly associated with metabolic health. Moderate intensity physical activity was weakly related to health, and sedentary time and light physical activity were not related to health. Conclusions: This study is the first to determine the multivariate physical activity signature related to metabolic health in children across the whole intensity spectrum. This novel approach shows that vigorous physical activity is strongest related to metabolic health. We recommend future studies adapt a multivariate analytic approach to further develop the field of physical activity epidemiology.

\section{Commentary}

Accelerometry-based activity monitors are an objective and valid measure of physical activity, providing high resolution timestamped data. To give biological meaning to accelerometer output, physical activity is frequently reported as time spent at a given activity intensity (eg, moderate-to-vigorous physical activity [MVPA]) using accelerometer cut-points.

The problems with using cut-points to quantify physical activity are well documented, but in brief include: (a) cut-points are wearsite-, and population- (eg, age-group) specific, leading to results that are not comparable across studies $(22,136,160)$; (b) 2 participants with similar levels of activity may score very differently if 1 has activity falling just above a cut-point and 1 has activity falling just below the cut-point; (c) sometimes participants fail to obtain any activity above cut-points (particularly in the vigorous range), consequently scoring zero. Also, as only approximately $5 \%$ of children's time is spent in MVPA, use of cut-points often means most of the accelerometer data is simply thrown away. Further, analyzing data using cut-points presupposes the activity intensities that may be pertinent for health, rather than letting the data determine the aspects of physical activity that are most highly associated with health.

Aadland and colleagues (3) present an innovative solution to these problems by applying multivariate pattern analysis to accelerometer data. This allowed them to investigate associations between the whole spectrum of physical activity intensities to determine the multivariate physical activity signature associated with metabolic health in their sample of 841 children, aged 10 years. The multivariate physical activity signature has the notable advantage of covering the whole range of accelerometer intensities in incremental steps, thus allowing identification of which intensities of activity are associated with health and where associations are strongest. Importantly, the approach is data driven. Given increasing evidence for high-intensity activity to be most strongly associated with health in adults (82; Rowlands et al, unpublished) and children (Rowlands et al, unpublished), focusing only on predetermined intensities potentially limits research into physical activity and health. A barrier to investigating the whole spectrum of physical activity intensities has been the strong multicollinearity between the variables, which cannot be handled by common statistical methods. However, the multivariate pattern analysis approach used by Aadland et al (3) can. Like other statistical methods recently applied to accelerometer data analysis (eg, 69, 105) it is commonly used in other disciplines, but not in physical activity research.

Results from this study highlight the importance of higherintensity physical activity for individual risk factors for metabolic health and on overall composite risk score. Significantly, physical activity intensities between 5,000 and 6,999 ActiGraph counts per minute (cpm) were most strongly associated with health; notably, this is higher than intensity cut-points frequently used for moderate and vigorous physical activity (eg, the cut-points for moderate and vigorous intensity using Evenson cut-points are 2,296 and 4,012 cpm, respectively [51]). As the authors point out, this suggests that future recommendations for children's physical activity may need to increase the focus on vigorous-intensity activity. This study focuses on children aged 10 years and is cross-sectional; an important next step will be to apply the method to different populations (eg, younger and older children) and to longitudinal data to determine the predictive performance of the associations shown. While this study focused on waist-worn ActiGraph data collected during a waking wear protocol and metabolic health, the methods could be applied to any of the many accelerometer datasets being collected worldwide, irrespective of wear site or whether the protocol is 24-hour wear or waking wear, and for any health outcome.

With higher-intensity activity, there are greater returns for a given duration of activity. This is recognized in current adult physical activity recommendations, which state 150 minutes per week of MVPA or 75 minutes of VPA (or combination). This tradeoff is illustrated nicely in Figure 3 of the article for incremental intensity ranges (3). In fact, the relative importance of a given duration of time spent in vigorous-intensity physical activity for metabolic health was 5 times greater than for the same amount of time spent in moderate-intensity physical activity; this is a much greater added benefit of higher-intensity activity than suggested by current physical activity guidelines. There have been calls for the development of future physical activity guidelines to be derived directly from accelerometer data (160). Perhaps multivariate pattern analysis could provide an evidence base to develop physical activity guidelines informed by accelerometer data that also reflect the intensity/duration trade-off in associations between physical activity and health.

\section{References}

1. 2018 Physical Activity Guidelines Advisory Committee. 2018 Physical Activity Guidelines Advisory Committee Scientific Report. Washington, DC: U.S. Department of Health and Human Services; 2018.

2. Aadland E, Anderssen SA, Andersen LB, et al. Aerobic fitness thresholds to define poor cardiometabolic health in children and 
youth. Scand J Med Sci Sport. 2018; Epub ahead of print. doi: 10. 1111/sms. 13330

3. Aadland E, Kvalheim OM, Anderssen SA, Resaland GK, Andersen LB. The multivariate physical activity signature associated with metabolic health in children. Int J Behav Nutr Phys Act. 2018; 15:77. doi: 10.1186/s12966-018-0707-z

4. Abderrahman AB, Rhibi F, Ouerghi N, Hackney AC, Saeidi A, Zouhal $\mathrm{H}$. Effects of recovery mode during high intensity interval training on glucoregulatory hormones and glucose metabolism in response to maximal exercise. J Athl Enhanc 2018;7(3):292.

5. Agbaje AO, Haapala EA, Lintu N, et al. Peak oxygen uptake cutpoints to identify children at increased cardiometabolic risk - The PANIC Study. Scand J Med Sci Sports. 2019;29(1):16-24. doi: 10. 1111/sms. 13307

6. Ahmaidi S, Granier P, Taoutaou Z, Mercier J, Dubouchaud H, Prefaut C. Effects of active recovery on plasma lactate and anaerobic power following repeated intensive exercise. Med Sci Sports Exerc. 1996;28(4):450-456.

7. Álvarez-Bueno C, Pesce C, Cavero-Redondo I, Sánchez-López M, Martínez-Hortelano JA, Martínez-Vizcaíno V. The effect of physical activity interventions on children's cognition and metacognition: A systematic review and meta-analysis. J Am Acad Child Adolesc Psychiatry. 2017;56:729-738.

8. Armstrong N, Barker AR. Endurance training and elite young athletes. In: Armstrong N, McManus AM, eds. The Elite Young Athlete. Basel, Switzerland: Karger; 2011.

9. Armstrong N, Welsman J. Sex-specific longitudinal modeling of youth peak oxygen uptake. Pediatr Exerc Sci. 2018; Epub ahead of print. doi: 10.1123/pes.2018-0175

10. Armstrong N, Welsman J. Twenty-metre shuttle run: (mis)representation, (mis)interpretation, and mis(use). Br J Sports Med. 2018; Epub ahead of print. doi: 10.1136/bjsports-2018-100082

11. Armstrong N. Development of the Youth Athlete. Oxford, UK: Routledge; 2018.

12. Armstrong N. Paediatric physical activity and aerobic fitness. In: Draper N, Stratton G, eds. Physical Activity: A Multi-Disciplinary Approach. Oxford, UK: Routledge; 2018.

13. Arsa G, Lanza FC, Cambri LT, et al. Predicted equation for $\mathrm{VO}_{2}$ based on a 20-meter multistage shuttle run test for children. Int $J$ Sports Med. 2018;39(14):1049-1054. doi: 10.1055/a-0665-4700

14. Askling C, Karlsson J, Thorstensson A. Hamstring injury occurrence in elite soccer players after preseason strength training with eccentric overload. Scand J Med Sci Sports. 2003;13(4):244-250.

15. Astrand PO, Eriksson BO, Nylander I, Engstroem L, Karlberg P, Saltin B, Thoren C. Girl swimmers. With special reference to respiratory and circulatory adaptation and gynaecological and psychiatric aspects. Acta Paediatr Suppl. 1963;SUPPL147:1-75.

16. Aubert S, Barnes JD, Abdeta C, et al. Global Matrix 3.0 Physical activity report card grades for children and youth: Results and analysis from 49 countries. J Phys Act Health. 2018;15(S2): S251-S273. doi: 10.1123/jpah.2018-0472

17. Augustsson S, Ageberg E. Weaker lower extremity muscle strength predicts traumatic knee injury in youth female but not male athletes. BMJ Open Sport Exerc Med. 2017;3:e00222.

18. Barlow SE, Expert Committee. Expert committee recommendations regarding the prevention, assessment, and treatment of child and adolescent overweight and obesity: Summary report. Pediatrics. 2007;120(Suppl 4):S164-S192.

19. Best JR. Effects of physical activity on children's executive function: Contributions of experimental research on aerobic exercise. Dev Rev. 2010;30(4):331-551.
20. Bonewald L. Use it or lose it to age: A review of bone and muscle communication. Bone. 2019;120:212-218.

21. Bovard JM, Welch JF, Houghton KM, et al. Does competitive swimming affect lung growth? Physiol Rep. 2018;6(15):e13816.

22. Brazendale K, Beets MW, Bornstein DB, et al. Equating accelerometer estimates among youth: The Rosetta Stone 2. J Sci Med Sport. 2016;19(3):242-249.

23. Brener ND, Kann L, McManus T, et al. Reliability of the 1999 youth risk behavior survey questionnaire. J Adolesc Health. 2002;31(4): 336-342.

24. Bruni O, Ottaviano S, Guidetti V, et al. The Sleep Disturbance Scale for Children (SDSC). Construction and validation of an instrument to evaluate sleep disturbances in childhood and adolescence. J Sleep Res. 1996;5(4):251-261.

25. Bruunsgaard H, Pedersen BK. Special feature for the Olympics: Effects of exercise on the immune system: Effects of exercise on the immune system in the elderly population. Immunol Cell Biol. 2000;78(5):523-531.

26. Buchan DS, Knox G, Jones AM, Tomkinson GR, Baker JS. Utility of international normative $20 \mathrm{~m}$ shuttle run values for identifying youth at increased cardiometabolic risk. J Sports Sci. 2019;37(5):507-514. doi:10.1080/02640414.2018.1511318.

27. Cadegiani FA, Kater CE. Hormonal aspects of overtraining syndrome: a systematic review. BMC Sports Sci Med Rehabil. 2017; 9:14

28. Cadegiani FA, Kater CE. Hormonal response to non-exercise stress test in athletes with overtraining syndrome: Results from the Endocrine and metabolic Responses on Overtraining Syndrome (EROS)EROS-STRESS. J Sci Med Sport. 2018;21(7):648-653.

29. Cadenas-Sanchez C, Intemann T, Labayen I, et al. Physical fitness reference standards for preschool children: The PREFIT project. $J$ Sci Med Sport. 2018; Epub ahead of print. doi: 10.1016/j.jsams.2018. 09.227 .

30. Castro-Piñero J, Perez-Bey A, Cuenca-Garcia M, et al. Muscle fitness cut points for early assessment of cardiovascular risk in children and adolescents. J Pediatr. 2018; Epub ahead of print.

31. Chen JJ, Gendy E, Leu S, et al. Age, sex and weight effects on lactate and leukocyte response to exercise in children and adolescents. Eur J Sports Exerc Sci. 2018;6(1):1-10.

32. Coates BM, Staricha KL, Wiese KM, Ridge KM. Influenza A virus infection, innate immunity, and childhood. JAMA Pediatr. 2015; 169(10):956-963.

33. Cooper AR, Goodman A, Page AS, et al. Objectively measured physical activity and sedentary time in youth: The International Children's Accelerometry Database (ICAD). Int J Behav Nutr Phys Act. 2015;12:113.

34. Corder K, Sharp SJ, Atkin AJ, et al. Change in objectively measured physical activity during the transition to adolescence. $\mathrm{Br} J$ Sports Med. 2015;49(11):730-736.

35. Costigan SA, Eather N, Plotnikoff RC, Taaffe DR, Lubans DR. High-intensity interval training for improving health-related fitness in adolescents: A systematic review and meta-analysis. Br J Sports Med. 2015;49(19):1253-1261. doi: 10.1136/bjsports-2014-094490.

36. Cullati S, Kliegel M, Widmer E. Development of reserves over the life course and onset of vulnerability in later life. Nat Hum Behav. 2018;2:551-558.

37. Cumming SP, Brown DJ, Mitchell S, et al. Premier League academy soccer players' experiences of competing in a tournament bio-banded for biological maturation. J Sports Sci. 2018;36(7):757-765.

38. Delp MD. Differential effects of training on the control of skeletal muscle perfusion. Med Sci Sports Exerc. 1998;30(3):361-374. 
39. Department of Health and Children, Health Service Executive. The National Guidelines on Physical Activity for Ireland. Dublin, Ireland: Author; 2009.

40. Diamond A, Ling DS. Aerobic-exercise and resistance-training interventions have been among the least effective ways to improve executive functions of any method tried thus far. Dev Cogn Neurosci. 2018; Epub ahead of print.

41. Diamond A, Ling DS. Conclusions about interventions, programs, and approaches for improving executive functions that appear justified and those that, despite much hype, do not. Dev Cogn Neurosci. 2016;18:34-48.

42. Diamond A, Ling DS. Fundamental questions surrounding efforts to improve executive functions (including working memory). In: Bunting M, Novick J, Dougherty M, Engle RW, eds. An integrative approach to cognitive and working memory training: Perspectives from psychology, neuroscience, and human development. New York, NY: Oxford University Press; In press.

43. Donnelly JE, Hillman CH, Castelli D, et al. Physical activity, fitness, cognitive function, and academic achievement in children: A systematic review. Med Sci Sports Exerc. 2016;48(6):1223-1224.

44. Draper N, Bird EL, Coleman I, Hodgson C. Effects of active recovery on lactate concentration, heart rate and RPE in climbing. J Sports Sci Med. 2006;5(1):97-105.

45. Drumm ML, Konstan MW, Schluchter MD, et al. Genetic modifiers of lung disease in cystic fibrosis. $N$ Engl J Med. 2005;353(14): 1443-1453.

46. Duppen N, Geerdink LM, Kuipers IM, et al. Regional ventricular performance and exercise training in children and young adults after repair of tetralogy of Fallot: Randomized controlled pilot study. Circ Cardiovasc Imaging. 2015;8(4):e002006.

47. Duppen N, Kapusta L, de Rijke YB, et al. The effect of exercise training on cardiac remodelling in children and young adults with corrected tetralogy of Fallot or Fontan circulation: A randomized controlled trial. Int J Cardiol. 2015;179:97-104.

48. Duppen N, Takken T, Hopman MT, et al. Systematic review of the effects of physical exercise training programmes in children and young adults with congenital heart disease. Int J Cardiol. 2013; 168(3):1779-1787.

49. Eddolis WTB, McNarry MA, Stratton G, Winn CON, Mackintosh KA. High-intensity interval training interventions in children and adolescents: A systematic review. Sports Med. 2017;47(11): 2363-2374. doi: 10.1007/s40279-017-0753-8.

50. Eliakim A, Schwindt C, Zaldivar F, Casali P, Cooper DM. Reduced tetanus antibody titers in overweight children. Autoimmunity. 2006; 39(2):137-141.

51. Evenson KR, Catellier DJ, Gill K, Ondrak KS, McMurray RG. Calibration of two objective measures of physical activity for children. J Sports Sci. 2008;26(14):1557-1565. doi:10.1080/02640410 802334196.

52. Faigenbaum AD, Kraemer WJ, Blimkie CJ, et al. Youth resistance training: Updated position statement paper from the national strength and conditioning association. J Strength Cond Res. 2009; 23(5 Suppl):S60-S79.

53. Faigenbaum AD, MacDonald JP. Dynapenia: It's not just for grownups anymore. Acta Paediatr. 2017;106(5):696-697.

54. Faigenbaum AD, Myer GD. Resistance training among young athletes: safety, efficacy and injury prevention effects. Br J Sports Med. 2010;44(1):56-63.

55. Faigenbaum AD, Rial Rebullido T, MacDonald JP. The unsolved problem of paediatric physical inactivity: It's time for a new perspective. Acta Paediatr. 2018;107(11):1857-1859.
56. Falk B, Dotan R. Measurement and interpretation of maximal aerobic power in children. Pediatr Exerc Sci. 2018; Epub ahead of print. doi: 10.1123/pes.2018-0191

57. Falk B, Klentrou P, Armstrong N, Rowland T, Kemper HCG. A brief history of pediatric exercise physiology. Pediatr Exerc Sci. 2018;30(1):1-10. doi: 10.1123/pes.2017-0246.

58. Farooq MA, Parkinson KN, Adamson AJ, et al. Timing and decline in physical activity in childhood and adolescence: Gateshead Millennium Cohort Study. Br J Sports Med. 2018;52(15):1002-1006.

59. Fédération Internationale de Football Association (FIFA). 265 million playing football. Retrieved from https://www.fifa.com/mm/ document/fifafacts/bcoffsurv/emaga_9384_10704.pdf. Accessed January 11, 2019.

60. Figueiredo AJ, Silva MJC, Cumming SP, Malina RM. Size and maturity mismatch in youth soccer players 11-to 14-years-old. Pediatr Exerc Sci. 2010;22(4):596-612.

61. Fraser BJ, Blizzard L, Tomkinson GR, et al. The great leap backward: Changes in the jumping performance of Australian children aged 11-12-years between 1985 and 2015. J Sports Sci. 2018; Epub ahead of print.

62. Fraser BJ, Huynh QL, Schmidt MD, Dwyer T, Venn AJ, Magnussen CG. Childhood muscular fitness phenotypes and adult metabolic syndrome. Med Sci Sports Exerc. 2016;48(9):1715-1722.

63. Fraser BJ, Schmidt MD, Huynh QL, et al. Tracking of muscular strength and power from youth to young adulthood: Longitudinal findings from the Childhood Determinants of Adult Health Study. J Sci Med Sport. 2017;20(10):927-931.

64. Gabler M, Prieske O, Hortobagyi T, Granacher U. The effects of concurrent strength and endurance training on physical fitness and athletic performance in youth: A systematic review and metaanalysis. Front Physiol. 2018;9:1057.

65. Galassetti PR, Nemet D, Pescatello A, Rose-Gottron C, Larson J, Cooper DM. Exercise, caloric restriction, and systemic oxidative stress. J Investig Med. 2006;54(2):67-75.

66. García-Hermoso A, Cavero-Redondo I, Ramírez-Vélez R, et al. Muscular strength as a predictor of all-cause mortality in an apparently healthy population: A systematic review and meta-analysis of data from approximately 2 million men and women. Arch Phys Med Rehabil. 2018;99(10):2100-2113.

67. Gharbi A, Masmoudi L, Chtourou H, Chaari N, Tabka Z. Effects of recovery mode on physiological and psychological responses and performance of specific skills in young soccer players. J Sports Med Phys Fitness. 2017;57(12):1590-1596.

68. Gibala MJ, Little JP, MacDonald MJ, Hawley JA. Physiological adaptations to low-volume, high-intensity interval training in health and disease. J Physiol. 2012;590(5):1077-1084.

69. Goldsmith J, Liu X, Jacobson JS, Rundle A. New insights into activity patterns in children, found using functional data analyses. Med Sci Sports Exerc. 2016;48(9):1723-1729.

70. Gomes TN, Dos Santos FK, Katzmarzyk PT, Maia J. Active and strong: physical activity, muscular strength, and metabolic risk in children. Am J Hum Biol. 2017;29(1):22904.

71. Granacher U, Lesinski M, Busch D, et al. Effects of resistance training in youth athletes on muscular fitness and athletic performance: A conceptual model for long-term athlete development. Front Physiol. 2016;7:164.

72. Gu Y, Harley IT, Henderson LB, et al. 2009. Identification of IFRD1 as a modifier gene for cystic fibrosis lung disease. Nature. 2009; 458(7421):1039-1042.

73. Gunnell KE, Poitras VJ, LeBlanc A, et al. Physical activity and brain structure, brain function, and cognition in children and youth: A 
systematic review of randomized controlled trials. Ment Health Phys Act. 2018; Epub ahead of print. doi: 10.1016/j.mhpa.2018.11.002

74. Hebestreit H, Arets HG, Aurora P, et al. Statement on exercise testing in cystic fibrosis. Respiration. 2015;90(4):332-351.

75. Hebestreit H, Kieser S, Rudiger S, et al. Physical activity is independently related to aerobic capacity in cystic fibrosis. Eur Respir J. 2006;28(4):734-739.

76. Henriksson H, Henriksson P, Tynelius P, Ortega FB. Muscular weakness in adolescence is associated with disability 30 years later: A population-based cohort study of 1.2 million men. Br J Sports Med. 2018; Epub ahead of print.

77. Hibbert M, Lannigan A, Raven J, Landau L, Phelan P. Gender differences in lung growth. Pediatr Pulmonol. 1995;19(2):129-134.

78. Hillman CH, McAuley E, Erickson KI, Liu-Ambrose T, Kramer AF. On mindful and mindless physical activity and executive function: A response to Diamond and Ling (2016). Dev Cogn Neurosci. 2018; Epub ahead of print.

79. Hulteen RM, Smith JJ, Morgan PJ, et al. Global participation in sport and leisure-time physical activities: A systematic review and metaanalysis. Prev Med. 2017;95:14-25.

80. Institute of Medicine (IOM). Fitness Measures and Health Outcomes in Youth. Washington, DC: The National Academies Press; 2012.

81. Jackowski SA, Faulkner RA, Farthing JP, Kontulainen SA, Beck TJ, Baxter-Jones AD. Peak lean tissue mass accrual precedes changes in bone strength indices at the proximal femur during the pubertal growth spurt. Bone. 2009;44(6):1186-1190.

82. Jelleyman C, Edwardson CL, Henson J, et al. Associations of increasing physical activity intensities with markers of insulin sensitivity. Med Sci Sports Exerc. 2017;49(12):2451-2458.

83. Joyner MJ, Casey DP. Regulation of increased blood flow (hyperemia) to muscles during exercise: a hierarchy of competing physiological needs. Physiol Rev. 2015;95(2):549-601.

84. Jürimäe J. Growth, maturation, and exercise during youth-The year that was 2017. Pediatr Exerc Sci. 2018;30(1):42-46. doi: 10.1123/ pes.2017-0291

85. Karachaliou F, Vlachopapadopoulou E, Psaltopoulou T, et al. Prevalence of asthma symptoms and association with obesity, sedentary lifestyle and sociodemographic factors: Data from the Hellenic National Action Plan for the assessment, prevention and treatment of childhood obesity (MIS301205). J Asthma. 2018; Epub ahead of print.

86. Koedijk JB, van Rijswijk J, Oranje WA, et al. Sedentary behaviour and bone health in children, adolescents and young adults: A systematic review-supplementary presentation. Osteoporos Int. 2017;28(10):3075-3076.

87. Lands LC, Heigenhauser GJ, Jones NL. Analysis of factors limiting maximal exercise performance in cystic fibrosis. Clin Sci (Lond). 1992;83(4):391-397.

88. Lang JE, Bunnell HT, Hossain MJ, et al. Being overweight or obese and the development of asthma. Pediatrics. 2018;142(6): $1-10$.

89. Lang JJ, Phillips EW, Orpana HM, et al. Field-based measurement of cardiorespiratory fitness to evaluate physical activity interventions. Bull World Health Organ. 2018;96(11):794-796.

90. Lang JJ, Tomkinson GR, Janssen I, et al. Making a case for cardiorespiratory fitness surveillance among children and youth. Exerc Sports Sci Rev. 2018;46(2):66-75.

91. Larsen MN, Nielsen CM, Wulff Helge E, et al. Positive effects on bone mineralisation and muscular fitness after 10 months of intense school-based physical training for children aged 8-10 years: the FIT FIRST randomised controlled trial. Br J Sports Med. 2018;52(4): 254-260.
92. Lee B, Adam A, Zenkov E, et al. Modeling the economic and health impact of increasing children's physical activity in the United States. Health Aff (Millford). 2017;36(5):902-908.

93. Lehmann M, Foster C, Keul J. Overtraining in endurance athletes: a brief review. Med Sci Sports Exerc. 1993; 25(7):854-862.

94. Leong DP, Teo KK, Rangarajan S, et al. Prognostic value of grip strength: findings from the Prospective Urban Rural Epidemiology (PURE) study. Lancet. 2015;386(9990):266-273.

95. Lesinski M, Prieske O, Granacher U. Effects and dose-response relationships of resistance training on physical performance in youth athletes: a systematic review and meta-analysis. $\mathrm{Br}$ J Sports Med. 2016;50(13):781-795.

96. Lloyd RS, Faigenbaum AD, Stone MH, et al. Position statement on youth resistance training: the 2014 International Consensus. $\mathrm{Br} \mathrm{J}$ Sports Med. 2014;48(7):498-505.

97. Lozano-Berges G, Matute-Llorente A, González-Agüero A, GómezBruton A, Gómez-Cabello A, Vicente-Rodríguez G, Casajús JA. Soccer helps build strong bones during growth: a systematic review and meta-analysis. Eur J Pediatr. 2018;177(3):295-310.

98. Lucas JA, Moonie S, Hogan MB, Evans WN. Efficacy of an exercise intervention among children with comorbid asthma and obesity. Public Health. 2018;159:123-128.

99. Lunt D, Briffa T, Briffa NK, Ramsay J. Physical activity levels of adolescents with congenital heart disease. J Physiother. 2003;49(1): 43-50.

100. Marcotte JE, Grisdale RK, Levison H, Canny GJ, Coates AL. Multiple factors limit exercise capacity in cystic fibrosis. Pediatr Pulmonol. 1986;2(5):274-281.

101. Marques A, Santos DA, Hillman CH, Sardinha LB. How does academic achievement relate to cardiorespiratory fitness, selfreported physical activity and objectively reported physical activity: a systematic review in children and adolescents aged 6-18 years. $\mathrm{Br} \mathrm{J}$ Sports Med. 2018;52(16):1039.

102. McGrath RP, Vincent BM, Lee IM, Kraemer WJ, Peterson MD. Handgrip strength, function, and mortality in older adults: A timevarying approach. Med Sc Sports Exerc. 2018;50(11):2259-2266.

103. McNarry AM, Jones A. The influence of training status on the aerobic and anaerobic responses to exercise in children: A review. Eur J Sport Sci. 2014;14(Suppl 1):557-568.

104. Meeusen R, Nederhof E, Buyse L, et al. Diagnosing overtraining in athletes using the two-bout exercise protocol. Br J Sports Med. 2010;44(9):642-648.

105. Millard LAC, Tilling K, Lawlor DA, Flach PA, Gaunt TR. Physical activity phenotyping with activity bigrams, and their association with BMI. Int J Epidemiol. 2017;46(6):1857-1860.

106. Milne N, Simmonds MJ, Hing W. A cross-sectional pilot study to examine the criterion validity of the modified shuttle test-paeds as a measure of cardiorespiratory fitness in children. Int J Environ Res Pub Health. 2018;15(10):2290. doi: 10.3390/ijerph15102290.

107. Mintjens S, Menting MD, Daams JG, van Poppel MNM, Roseboom TJ, Gemke RJBJ. Cardiorespiratory fitness in childhood and adolescence affects future cardiovascular risk factors: A systematic review of longitudinal studies. Sports Med. 2018;48(11):2577-2605. doi: 10.1007/s40279-018-0974-5.

108. Moran J, Clark CCT, Ramirez-Campillo R, Davies MJ, Drury B. (2018). A meta-analysis of plyometric training in female youth: Its efficacy and shortcomings in the literature. J Strength Cond Res. 2018; Epub ahead of print.

109. Moran J, Sandercock G, Ramirez-Campillo R, Clark CCT, Fernandes JFT, Drury B. A meta-analysis of resistance training in female youth: Its effect on muscular strength, and shortcomings in the literature. Sports Med. 2018;48(7):1661-1671. 
110. Morgado JP, Monteiro CP, Matias CN, et al. Long-term swimming training modifies acute immune cell response to a high-intensity session. Eur J Appl Physiol. 2018;118(3):573-583.

111. Murali M, Hofman PL, Derraik JGB, Cutfield WS, Hornung T, Gusso S. Exercise capacity and cardiac function in adolescents born post-term. Sci Rep. 2018;8(1):12963.

112. Nadeau KJ, Regensteiner JG, Bauer TA, et al. Insulin resistance in adolescents with type 1 diabetes and its relationship to cardiovascular function. J Clin Endocrinol Metab. 2010;95(2):513-521.

113. Nemet D, Meckel Y, Bar-Sela S, Zaldivar F, Cooper DM, Eliakim A. Effect of local cold-pack application on systemic anabolic and inflammatory response to sprint-interval training: a prospective comparative trial. Eur J Appl Physiol. 2009;107:411.

114. Nemet D, Oren S, Pantanowitz M, Eliakim A. Effects of a multidisciplinary childhood obesity treatment intervention on adipocytokines, inflammatory and growth mediators. Horm Res Paediatr. 2013;79(6):325-332.

115. Nixon PA, Orenstein DM, Kelsey SF, Doershuk CF. The prognostic value of exercise testing in patients with cystic fibrosis. $N$ Engl $J$ Med. 1992;327:1785-1788.

116. Ortega FB, Ruiz JR, Castillo MJ, Sjostrom M. Physical fitness in children and adolescence: a powerful marker of health. Int J Obesity. 2008;32:1-11.

117. Ortega FB, Silventoinen K, Tynelius P, Rasmussen F. Muscular strength in male adolescents and premature death: cohort study of one million participants. BMJ. 2012;345:e7279.

118. ParticipACTION. The Brain + Body Equation: Canadian kids need active bodies to build their best brains. The 2018 ParticipACTION Report Card on Physical Activity for Children and Youth. Toronto, Canada: ParticipACTION; 2018. Retrieved from: https:// participaction.cdn.prismic.io/participaction\%2F38570bed-b325-4fc88855-f15c9aebac12_2018_participaction_report_card_-_full_report_ $0 . p d f$

119. Pedersen BK, Ostrowski K, Rohde T, Bruunsgaard H. Nutrition, exercise and the immune system. Proc Nutr Soc. 1998;57(1):43-47.

120. Pena-Gonzalez I, Fernandez-Fernandez J, Moya-Ramon M, Cervello E. Relative age effect, biological maturation, and coaches' efficacy expectations in young male soccer players. Res $Q$ Exerc Sport. 2018;89:373-379.

121. Peterson MD, Gordon PM, Smeding S, Visich P. Grip strength is associated with longitudinal health maintenance and improvement in adolescents. J Pediatr. 2018;202:226-230.

122. Pfeiffer K, Lobelo F, Ward DS, Pate RR. Endurance trainability of children and youth. In: Hebestreit $\mathrm{H}$, Bar-Or O, eds. The young athlete. Oxford, UK: Blackwell; 2008: 84-95.

123. Pianosi P, Leblanc J, Almudevar A. Peak oxygen uptake and mortality in children with cystic fibrosis. Thorax. 2005;60:50-54.

124. Piercy KL, Troiano RP, Ballard RM, et al. The physical activity guidelines for Americans. JAMA. 2018;320(19):2020-2028.

125. Pinedo-Villanueva R, Westbury LD, Syddall HE, et al. Health care costs associated with muscle weakness: A UK population-based estimate. Calcif Tissue Int. 2018; Epub ahead of print. doi: 10. 1007/s00223-018-0478-1

126. Quatman-Yates CC, Quatman CE, Meszaros AJ, Paterno MV, Hewett TE. A systematic review of sensorimotor function during adolescence: a developmental stage of increased motor awkwardness? Br J Sports Med. 2012;46:649-655.

127. Radtke T, Hebestreit H, Gallati S, et al. CFTR genotype and maximal exercise capacity in cystic fibrosis. A cross-sectional study. Ann Amer Thoracic Soc. 2018;15(2):209-216.

128. Ramírez-Vélez R, Peña-Ibagon JC, Martínez-Torres J, et al. Handgrip strength cutoff for cardiometabolic risk index among Colombian children and adolescents: The FUPRECOL Study. Sci Rep. 2017;7:42622.

129. Rastogi D. Quantifying the contribution of obesity to incident childhood asthma: It's about time. Pediatrics. 2018;142(6):1-2.

130. Ratel S, Williams CA. Neuromuscular fatigue. In: Armstrong N, van Mechelen W, eds. Oxford Textbook of Children's Sport and Exercise Medicine. 3rd ed. Oxford, UK: Oxford University Press; 2017: 121-132.

131. Regitz-Zagrosek V. Sex and gender differences in health. Science \& Society Series on Sex and Science. EMBO Rep. 2012;13(7): 596-603. doi: 10.1038/embor.2012.87

132. Renz H, Holt PG, Inouye M, Logan AC, Prescott SL, Sly PD. An exposome perspective: Early-life events and immune development in a changing world. J Allergy Clin Immunol. 2017;140(1):24-40.

133. Rioux BV, Kuwornu P, Sharma A, Tremblay M, McGavock JM, Sénéchal M. Association between handgrip muscle strength and cardiometabolic z-score in children 6 to 19 years of age: Results from the Canadian health measures survey. Metab Syndr Relat Disord. 2017;15(7):379-384.

134. Rogol AD, Cumming SP, Malina RM. Biobanding: a new paradigm for youth sports and training. Pediatrics. 2018;142(5):e20180423.

135. Rowland TW. Assessing myocardial function. In: Rowland TW, ed. Cardiopulmonary exercise testing in children and adolescents. Champaign, IL: Human Kinetics; 2018.

136. Rowlands AV. Moving forward with accelerometer-assessed physical activity: Two strategies to ensure meaningful, interpretable, and comparable measures. Pediatr Exerc Sci. 2018;30(4):450-456. doi: 10.1123/pes.2018-0201

137. Ruiz JR, Cavero-Redondo I, Ortega FB, Welk GJ, Andersen L, Martinez-Vizcaino V. Cardiorespiratory fitness cut points to avoid cardiovascular disease risk in children and adolescents; what level of fitness should raise a red flag? A systematic review and metaanalysis. Br J Sports Med. 2016;50(23):1451-1458.

138. Sandercock GRH, Cohen DD. Temporal trends in muscular fitness of English 10-year-olds 1998-2014: An allometric approach. J Sci Med Sport. 2019;22(2):201-205.

139. Schonfeld-Warden N, Warden CH. Pediatric obesity. An overview of etiology and treatment. Pediatr Clin North Am. 1997;44(2):339-361.

140. Schutte NM, Nederend I, Hudziak JJ, Bartels M, de Geus EJ. Twinsibling study and meta-analysis on the heritability of maximal oxygen consumption. Physiol Genom. 2016;48(3):210-219.

141. Schwarzfischer P, Gruszfeld D, Socha P, et al. Longitudinal analysis of physical activity, sedentary behaviour and anthropometric measures from ages 6 to 11 years. Int J Behav, Nutr Phys Act. 2018; 15:126.

142. Selvadurai HC, McKay KO, Blimkie CJ, Cooper PJ, Mellis CM, Van Asperen PP. The relationship between genotype and exercise tolerance in children with cystic fibrosis. Am J Respir Crit Care Med. 2002;165(6):762-765.

143. Sharif I, Wills TA, Sargent JD. Effect of visual media use on school performance: A prospective study. J Adolesc Health. 2010;46(1): 52-61.

144. Sherrill DL, Camilli A, Lebowitz MD. On the temporal relationships between lung function and somatic growth. Am Rev Respir Dis. 1989;140(3):638-644.

145. Simon G, Reid L, Tanner JM, Goldstein H, Benjamin B. Growth of radiologically determined heart diameter, lung width, and lung length from 5-19 years, with standards for clinical use. Arch Dis Child. 1972;47(253):373-381.

146. Singh AS, Saliasi E, van den Berg V, et al. Effects of physical activity interventions on cognitive and academic performance in children and adolescents: A novel combination of a systematic review and 
recommendations from an expert panel. Br J Sports Med. 2018; Epub ahead of print.

147. Slimani M, Paravlic A, Granacher U. A meta-analysis to determine strength training related dose-response relationships for lower-limb muscle power development in young athletes. Front Physiol. 2018;9:1155. doi: 10.3389/fphys.2018.01155.

148. Smith JJ, Eather N, Morgan P, Plotnikoff R, Faigenbaum AD, Lubans DR. The health benefits of muscular fitness for children and adolescents: A systematic review and meta-analysis. Sports Med. 2014;44(9):1209-1223.

149. Smith PJ, Blumenthal JA, Hoffman BM, et al. Aerobic exercise and neurocognitive performance: A meta-analytic review of randomized controlled trials. Psychosom Med. 2010;72(3):239-52.

150. Somarriba G, Extein J, Miller TL. Exercise rehabilitation in pediatric cardiomyopathy. Prog Pediatr Cardiol. 2008;25(1):91-102.

151. Sommi C, Gill F, Trojan JD, Mulcahey MK. Strength and conditioning in adolescent female athletes. Phys Sportsmed. 2018;46(4): 420-426.

152. Stavnsbo M, Resaland GK, Anderssen SA, et al. Reference values for cardiometabolic risk scores in children and adolescents: Suggesting a common standard. Atheroscler. 2018;278:299-306.

153. Tan VPS, Macdonald HM, Kim S, et al. Influence of physical activity on bone strength in children and adolescents: a systematic review and narrative synthesis. J Bone Miner Res. 2014;29(10): 2161-2181.

154. Tanner JM. Fallacy of per-weight and per-surface area standards and their relation to spurious correlation. J Appl Physiol. 1949;2(1): $1-15$.

155. Timmons BW. Paediatric exercise immunology: health and clinical applications. Paed Exerc Immunol. 2005;11:108-144.

156. Timmons BW, Tarnopolsky MA, Snider DP, Bar-Or O. Immunological changes in response to exercise: influence of age, puberty, and gender. Med Sci Sports Exerc. 2006;38(2):293-304.

157. Tolfrey K, Smallcombe J. High-intensity interval training. In: Armstrong N, van Mechelen W, eds. Oxford Textbook of Children's Sport and Exercise Medicine. 3rd ed. Oxford, UK: Oxford University Press; 2017:477-491.

158. Tremblay MS, Carson V, Chaput J-P, et al. Canadian 24-hour movement guidelines for children and youth: an integration of physical activity, sedentary behaviour, and sleep. Appl Physiol Nutr Metab. 2016;41(6, Suppl 3):S311-S327.

159. Tremblay MS, Warburton DER, Janssen I, et al. New Canadian physical activity guidelines. Appl Physiol Nutr Metab. 2011;36(1): 3647-4658.

160. Troiano RP, McClain JJ, Brychta RJ, Chen KY. Evolution of accelerometer methods for physical activity research. Br J Sports Med. 2014;48(13):1019-1023.
161. US Department of Health and Human Services. Physical Activity Guidelines for Americans, 2nd ed. Washington, DC: Author; 2018.

162. Vaitkeviciute D, Lätt E, Mäestu J, et al. Physical activity and bone mineral accrual in boys with different body mass parameters during puberty: a longitudinal study. PLoS One. 2014;9:e107759.

163. van de Weert-van Leeuwen PB, Slieker MG, Hulzebos HJ, Kruitwagen CLJJ, van der Ent CK, Arets HGM. Chronic infection and inflammation affect exercise capacity in cystic fibrosis. Eur Respir J. 2012; 39(4):893-898.

164. Wagner PD. Why doesn't exercise grow the lungs when other factors do? Exerc Sport Sci Rev. 2005;33(1):3-8.

165. Walker G, Stracciolini A, Faigenbaum A, Myer G. Physical inactivity in youth. Can exercise deficit disorder alter the way we view preventive care? ACSM Health Fit J. 2018;22(2):42-46.

166. Walsh JJ, Barnes JD, Cameron JD, et al. Associations between 24 hour movement behaviours and global cognition in US children: a cross-sectional observational study. Lancet Child Adolesc Health. 2018;2(11):783-791.

167. Weaver CM, Gordon CM, Janz KF, et al. The National Osteoporosis Foundation's position statement on peak bone mass development and lifestyle factors: A systematic review and implementation recommendations. Osteopor Int. 2016;27(4):1281-1386.

168. Welsman J, Armstrong N. Interpreting ratio scaling in youth: The fallacy of ratio scaling. Pediatr Exerc Sci. 2018; Epub ahead of print. doi:10.1123/pes.2018-0141.

169. Welsman JR, Armstrong N. Interpreting exercise performance data in relation to body size. In: Armstrong N, van Mechelen W, eds. Paediatric exercise science and medicine. 2nd ed. Oxford, UK: Oxford University Press; 2008: 13-22.

170. Winter EM, Nevill AM. Scaling: Adjusting for differences in body size. In: Eston R, Reilly T, eds. Kinanthropometry and exercise physiology laboratory manual: Tests, procedures and data. 3rd ed. Oxford, UK: Routledge; 2009: 300-320.

171. Wittekind SG, Gerdes Y, Mays W, Chin C, Jefferies JL. Cardiac rehabilitation improves cardiometabolic health in young patients with nonischemic dilated cardiomyopathy. Texas Heart Inst J. 2018; 45(1):27-30.

172. Woods JA, Davis JM, Smith JA, Nieman DC. Exercise and cellular innate immune function. Med Sci Sports Exerc. 1999;31(1):57-66.

173. World Health Organization (WHO). Global Action Plan on Physical Activity 2018-2030: More Active People for a Healthier World. Geneva, Switzerland: Author; 2018.

174. World Health Organization (WHO). Global Recommendations on Physical Activity for Health. Geneva, Switzerland: Author; 2010.

175. Wright LA, Hirsch IB. Metrics beyond hemoglobin A1C in diabetic management: time in range, hypoglycemia, and other parameters. Diabetes Technol Ther. 2017;19(S2):S16-S26. 